\title{
Zur Verallgemeinerung des Vierscheitelsatzes und seiner Umkehruug.
}

Herin Wilhelm Blaschise zum 60. Geburtstag gewidmet.

Von OTro Haupt (in Erlangen).

\section{Einleitung.}

1.1. Bekannt ist folgender Satz (1):

(1) Wird ein stetig gekrtimmtes ( $\left.{ }^{2}\right)$ Oval 0 von einem Kreis in $2 n$ Punkten $(n \geq 2)$ getroffen, so besitzt 0 mindestens $2 n$ Scheitel.

Dabei wird hier $\left({ }^{3}\right)$ als Scheitel jeder Punkt $P_{\varepsilon} 0$ bezeichnet, dessen zyklischer Ordnungswert mindestens Vier ist $(d . h$. jeder Punkt, in dessen beliebig kleiner Umgebung mehr als drei auf einem Kreis gelegener Punkte von 0 existieren). Di jedes Oval mit geeigneten Kreisen (mindestens) vier Punkte gemeinsam hat, folgt aus (1) speziell der ( $\left.{ }^{4}\right)$

Vierscheitelsatz: Jedes stetig gekrümmte Oval besitzt mindestens vier Scheitel.

Übrigens kann (1) auch so ausgesprochen werden :

(1) Jedes stetig gekrummte Oval, dessen zyklischer Ordnungswert mindestens $2 n$ betrigt, besitzt mindestens $2 n$ Scheitel.

(1) Brascme, W., Kreis und Kugel, Loipzig 1916, S. 161; MukropaibHyaya, S., (I) Some general theorems in the geometry of a plane curve; "Calcutta University Publications I922 = Collected geometrical Papers ", Part. I (Calcutta 1929), S. 118; ferner (II) Extended minimum. number theorems of cyctic and sextactic points on a plane convex oval, . Math. Zeitschr", 33 (1931), S. 648 ff.

(2) Die hier (Nr. 1.1. und 1.2.) betrachteten Ovale bzw. Konvexbogen sollen zwoimal stetig differonzierbar sein und iiberall endliche, von Null vorsehiedeno Krtimmung besitzen; das soll heissen: Das Oval gestattet eine Darstollung $x=f(t), y=g(t), 0 \leq t \leq 1$, wobei $f$ und $g$ zweimal stetig diffenzierbar sind mit $x^{\prime \prime} y^{\prime}-x^{\prime} y^{\prime \prime} \neq 0$. Wir bozeichnen ein solches Oval kurz als stetig gekrummt.

(3) In der klassischen Differentialgeometrie wird als "Scheitel * jeder Punkt des Ovals erklürt, der eine Extremstelle für den Krïmmungsradius, d. h. für den Radius des (unter unserer Differenzierbarkeitsvoraussetzung existierenden) freien Krümmungskreises (vgl. im Text Nr. 1.3.) liefert. In dem fitr uns in Betracht kommenden Falle, dass die Scheitel im Sinne der oben im Text gegebenen Definition isoliert liegen, sind beide Definitionen äqui. valent; vgl. HAUт, $Z M^{*}$ geometrisehen Kennzeichnung der Scheitel ebener Kurven, *Arohiv. d. Math. *, 1. (1948), S. 102 ff.

(1) MUKHOPADIYAYA, S, (I) New methods in the gemetry of a plane arc: I. Cyclic and sextatic points, *Bull. Calcutta math. Soo., Vol. I. (1909) = Collected geometrical papers ", Part. I, S. 15, prop. III., sowie (II) a. a. O. ('); Braschke, W., (I) a. a. O. ('), S. 
1.2. Weniger bekannt als (1) scheint der folgende Satz $\left({ }^{5}\right)$ zu sein :

(P) Jeder stetig gekriummte Konvexbogen vom zyklischen Ordnungswert Vier besitzt höchstens vier Scheitel, woraus zusammen mit (1) noch folgt:

(3) Ein stetig gekrümmtes Oval ist vom zyklischen Ordnungswert Vier dann und nur dann, wenn es genau vier Scheitel besitzt $\left({ }^{6}\right)$.

1.3. Noch weniger bekannt dïrften die in Nr. 2.2. formulierten weitgehenden Verallgemeinerungen sein, deren die in Nr. 1.1. und 1.2. angeführten Sätze fähig sind, Verallgemeinerungen nämlich in dem Sinne, dass man die Kreise ersetzen kann z. B. (7) durch Ellipsen, wobei dann entsprechend die Zahl Sechs an Stelle der Zahl Vier tritı, oder allgemein (7) durch Kurven K mit-grob gesprochen- der Eigenschaft, dass je zwei Kurven II höchstens $2 t$ Punkte gemeinsam haben ( $t$ fest mit $t \geq 1$ für alle $\mathbf{K}$ ) (Genaue Angabe der Voraussetzungen in $\mathrm{Nr}$. 2.1.). Für Satz (2) ist diese Verallgemeinerung unter sehr schwachen Annahmen uiber die Kurven $\mathbf{K}$ sowie uber die an Stelle der Konvexbogen tretenden Bogen bewiesen ( ${ }^{5}$ ). Demgegenüber setzen die uns bekannten Beweise der entsprechenden Verallgemeinerung von Satz (1) mehr voraus $\left({ }^{8}\right)$. Es soll deshalb ein diese Voraussetzungen vermeidender und zugleich, wie uns scheint, einfacherer $\left(^{9}\right)$ Beweis des verallgemeinerten Satzes $(1)$ angegeben werden, ferner ein, ebenfalls etwas vereinfachter, Beweis

160, sowie (IT) Vorlesungen über Differentialgeometrie, I., 3. Aufl., Berlin 1980, S. 30 ff. und die dort (S. 32) angegebene Literatur; dazu noch v. Sz. NAGY, G., Ein Beveis des Vierscheitelsatzes, «Jahresber. d. d. Math, Ver. ' 52 (1942), Abt. I, S. 198.200; Weitere Literatur hei Bonnesen.Fenchel, Theorie d. konvexen Körper, "Elgebnisse d. Math. u. Grenzgebiete", 范. Bd., Berdin 1985; Für nicht-konvexe Kurven vgl. H. Kneser,' Newer Beneis des Vierscheitel. satzes, Christ. Huygens Bd. 2 (1922-23), $315 \mathrm{ff}$.

(5) Vgl. HAuPT, Zur Theorie der Realitätsordnungen, “Monatsh. f. Math. u. Physik"g 40 (1983), S. 47.

(6) Jubi, C., Om simple cykliske kurver, "Danske Vidensk. Selskab Skrilter, 7. R., naturv. og matem. Afd." VIII, Nr. 6 (1911); Blaschkк, a. a. O. ( $\left.{ }^{4}\right)$, (II) S. 49, Aufg. 21 ; Bose, R. C., Circles of curvature, "Math. Keitschr, ", 352 (1932), S. 24; HAUPT, a. a. O. ("), S. 48; Bol, G., Ein Sate über Eilinien, "Abh. math. Seminar Hamburg ", 13 (1940), S. 319.

(7) Bezinglich Satz (1) sowie bezïglich des Vierscheitelsatzes, siche Muruopadryaya a. a. O. (1) und (4). - Anmerkung bei der Korrektur: Inzwischen jerschien noch G. BoL, Eitinien und sextaktische Punkte, "Archiv. d. Math. », 1 (1948), S. 94 ff., worin unser Sats (B) (1) in Nr. 2.2. fiir den speziellen Fall bewiesen wird, dass der Grundbogen $\mathbf{B}$ ein analytisches" Oval ist und dass die Ordnungscharakteristiken diejKegelschnitte sind. Die ausserdem dort $(\$ 8)$ gewonnenen Sutze dürften sich als Folgerungen aus unserem Satz (B) (1) fiir den 3 Fall eines Bogens $\mathbf{B}$ ergeben.

(8) Mukhopadhyaya a. a. O. (1) (I) und (II) je Nr. 1. und.2,

(7) Wir bedienen uns numlich der Zuriekführung auf den Fall $t=0$ (in unseror spïteren Bezeichnung $k=1$ ) vermittelst vollständiger Induktion, wodurch mancherlei Beweisschwierigkeiten vermejdbar worden. Dafür muss das Auftreten von Grundpunkten (vgl. Nr. 2.1.) auf dem Grundbogen zugelassen werden, was aber keinerlei Komplikationen mit sich bringi. 
von Satz (2. Damit ist dann auch Satz (3) entsprechend verallgemeinert. (Genaue Formulierung der Verallgemeinerungen in Nr. 2.2.).

Die im Falle der zyklischen Ordnung gemachten Differenzierbarkeitsannahmen $\left({ }^{2}\right)$ sind damit gquivalent $\left({ }^{\circ}\right)$, dass in jedem Punkt des Ovals der freie Krümmungskreis (eindentig) existiert (und endlichen, von Null verschiedenen Radius besitzt); bei den ins Auge gefassten Verallgemeinerungen entspricht dieser Differenzierbarkeitsannahme die Forderung der (eindeutigen) Existenz der "k-Paratingente $»$ in jedem Punkt (vgl. Nr. 2.1., IV.). Diese D iffer̀en. zierbarkeitsvorausetzung IV. ist mindestens für Satz (1) und (3) wesentlich, insofern ohne sie der Vierscheitelsatz und damit auch Satz (1) und (3) bzw, seine Verallgemeinerung nicht zu gelten braucht, wie einfache Beispiele zeigen (vgl. Nr. 3.2.1.2.). Setzt man aber die Existenz des freien Krümmungskreises nicht voraus (tuch nicht die der Tangente), so gilt doch wenigstens (vgl. Nr. 3.2.1.1.) der Satz:

(4) Jedes Oval, welches von einem Kreis in $(3 t+1)$ Pnnkten $(t \geqq 1)$ getroffen wird, besitzt mindestens $(t+1)$ Scheitel (gleichgültig, ob in jedem Punkt des Ovals der freie Krümmungskreis existiert oder nicht).

Als Spezialfall fiir $t=1$ ergibt sich daraus der

Zweischeitelsatz: Jedes Oval besitzt mindestens zwei Scheitel.

Die Beweisiberlegungen grïnden sich auf eine Weiterbildurg und Vertiefung der Methode vou Mukhopadhyaya ( $\left.{ }^{14}\right)$. Mit ihr lassen sich ubrigens auch (rein geométrisch) Verallgemeinerungen der Ovalsătze von Böhmer, Carleman und Mohrmann $\left({ }^{12}\right)$ gewinnen, sowie Verallge meinerungen anderer Kennzeichnungen $\left({ }^{13}\right)$ der Ovale vom zyklischen Ordnungswert Vier beweisen; ferner ist sie Hilfsmittel bei ordnungsgeometrischen Strukturuntersuchungen $\left({ }^{14}\right)$. Genauere Angaben über die beim Beweise der einzelnen Sätze jeweils benutzten Differenzierbarkeitsvoraussetzungen finden sich im Zusatz zu Nr. 2.2.

Es bedarf kaum der Hervorhebung, dass durch die nachstehenden Betrachtungen die in Rede stehencen Sätze als solche der direkten Infinitesimalgeometrie sich erweisen.

(10) Vgl. Haupt-Aumann.Panc, Differential-u. Integralvechnung, 2. Aufl., Berlin 1948, Ba. II., Nr. 2.2.6.1., Satz 1a.

(ii) MuKHOPADHYAYA, a. a. 0. (').

(12) Vgl. HAupt, Über Verallgemeinerungen des Böhmerschen u. vervandter Ovalsätze, "Abh. math. Seminar Humburg ", 15 (1943), S, 130 ff.

(i3) HaUP, Verallyemeinerung eines Satzes usw, "Sitz. Bor. d. medizin.-physikal. Sozietiit zu Erlangen ». 65 (1934), S. 279 ff., sowie ebenda 67 (1935), S. 13.

(14) Vgl. z. B. HAUPT, Zyklisch ordnungshomogene Bogen, "Journ. f. d. r. u. angew. Matlk. "; 178 (19:37), S. 14 ff. und 180 (1989), S. 44 ff. 


\section{Formulierung der verallgemeinerten Sätze.}

\subsection{Voraussetzungen.}

Wir stellen zunachst zusammen, was bei den nachstehenden Betrachtungen vorausgesetzt wird $\left({ }^{15}\right)$.

I. Grandbogen (Grundkurve). In einem einfach zusammenhän. genden Gebiet $\mathbf{G}$ (Grundgebiet) ${ }^{\left({ }^{6}\right)}$ der euklidischen Ebene $E_{2}$ sei ein einfacher abgeschlossener oder offener Bogen, der Grundbogen B, oder eine einfa. che, geschlossene (beschrinkte) Kurve, die Grundkurve $\mathbf{C}$ gegeben. Jeder Teilbogen von $\mathbf{B}$ oder $\mathbf{C}$ ist ebenfalls ein Grundbogen insofern namlich, als er den jetzt anzugebenden Voraussetzungen I-III. oder I.-IV. genügt. Es soll B bzw. C mit keiner der (sogleich zu erklarenden) Ordnungscharakteristiken Teilbogen gemeinsam haben. Wenn im Folgenden vom «Grundbogen» ohne weiteren Zusatz die Rede ist, so soll damit auch der Fall einer Grundkurve einbegriffen sein.

II. Ordnungsoharakteristiken. (a) In $\mathbf{G}$ ist ein System $\mathbf{k}$ von sogenannten $O r d n u n g s c h a r a k t e r i s t i k e n$, kurz $O C h$, gegeben der folgenden Art: Jede OCh ist entweder ein einfacher, offener Bogen, dessen (ev. zusammenfallende) Endpunkte der Begrenzung ron $\mathbf{G}$ angehören, oder eine einfache, geschlossene Kurve. - (b) Existenz und Eindeutigkeit der OCh. Durch $k$ verschiedene Punkte von $G$, die zu beliebigen verschiedenen Punkten des Grundbogens je hinreicliend benachbart sind, geht mindestens eine OCh. Es gibt höchstens endlich viele (ev. keine) (k-) Grundpunkte, d. h. Punkte, die allen OCh gemeinsam sind; sämtliche Gunndpunkte sollen auf dem Grundbogen liegen. Irgı nd zwei verschiedene OCh haben au:ser den Grundpunkten höchstens $(k-1)$ Punkte in $\mathbf{G}$ gemeinsam; dabei ist die sogenannte Grundzahl $k$ eine, durch das System $\mathbf{k}$ der OCh hestimmte, natürliche Zahl $\geq 1$. Fuir jede OCh $\mathbf{K}$ soll $\mathbf{B K}$ nirgends dicht liegen auf $\mathbf{B}$, es soll also kein $\mathbf{K}$ mit $\mathbf{B}$ einen Teilbrgen gemeinsam haben. - (c) Stetigkeit der Ordnungscharakteristiken. Sind $P_{j}^{\prime}, j=1, \ldots, k$, irgend $k$ untereinander und von den Grundpunkten verschiedene I'unkte des Grundbogens und ist $\mathbf{K}^{\prime}$ die, alle $P_{j}^{\prime}$ enthaltende OCh, so existiert zu jedem $\eta_{i}>0$ ein $\delta=\delta\left(\eta ; P_{1}^{\prime}, \ldots, P_{k}{ }^{\prime}\right)>0$ von folgender Eigenschaft: $\left(1^{\prime}\right)$ jede $O C h \mathbf{K}$, die nicht fremd ist je zur $\delta$-Umgebung eines jeden der $P_{j}^{\prime}$, liegt in der $\gamma_{j}$-Umgebung von $\mathbf{K}^{\prime}$; und umgekehrt liegt $\mathbf{K}^{\prime}$ in der $\eta$-Umgebung von K. - (2') Ist $\mathbf{T}^{\prime}$ ein Teilbogen von $\mathbf{K}^{\prime}$ mit den Begrenzungspunkten $Q^{\prime}, R^{\prime}$ auf $\mathbf{K}^{\prime}$, so gibt es in der $\delta$-Umgebung von $Q^{\prime}$ bzw. $R^{\prime}$ je einen Punkt $Q$ bzw. $R$ von $\mathbf{K}$ derart, dass $Q, R$ dir Endpunkte eines zu 'T' $\eta$-benachbarten Teillogens ' $\mathbf{T}$ von $\mathbf{K}$ sind. - (d) Verhalten in der Umgebung der Grundpunkte. Im Zusimmenhang mit der Stetigkeit der OCh fordern wir

(15) Zu Vor. 1 - III, vgl. HAu's, a. a. O. (5), Nr. 1.1.

(15) Das Grundgebiet $\left(\dot{3}\right.$ kann auch mit $F_{z}$ identisch sein; als Begrenaung von $(4$ gelte dann bie uneigentlichr Geradr der zur affinen Ebene arweiterten $E_{2}$. 
nch folgendes: (1) Jeder Grundpunkt soll «im allgemeinen» Schnittpunkt ${ }^{\left({ }^{17}\right)}$ von $\mathbf{B}$ mit den OCh sein, d. h. : ist der Grundpunkt $G$ Stützpunkt $\left({ }^{17}\right)$ der OCh $\mathbf{K}$ mit $\mathbf{B}$, so gibt es zu $\mathbf{K}$ beliebig benachbarte $0 \mathrm{Ch} \mathbf{K}^{\prime}$ derart, dass $G$ Schnittpunkt von $\mathbf{K}^{\prime}$ mit $\mathbf{B}$ ist. - (2) Wir werden es fast ausschliesslich mit dem Fall zu tun haben, dass die OCh nur endlich viele Punkte mit $\mathbf{B}$ gemeinsam haben; die auftretenden Schnitt-und Stutzpunkte sind dann stets isoliert. Ist die Anzahl der gemeinsamen Punkte von $\mathbf{B}$ mit den OCh endlich oder speziell beschrïnkt (also für jede $\mathrm{OCh} \mathbf{C}$ nicht grösser als eine (von $\mathbf{C}$ unabhängige) feste Zahl), so soll gelten: Es seien $Q_{1}, \ldots, Q_{k-1}$, feste, von den Grundpunkten verschiedene Punkte von B; ferner sei $G^{\prime}$ ein Grundpunkt und $\mathbf{U}^{\prime}$ eine, zu den ubrigen Grundpunkten sowie zu den $Q_{j}, j=1, \ldots, k-1$, fremde Umgebung von $G^{\prime}$ anf $\mathbf{B}$. Durch irgend einen, von $G^{\prime}$ verschiedenen Punkt $Q^{\prime} \varepsilon \mathbf{U}^{\prime}$ und durch die $Q_{1}, \ldots, Q_{k-1}$ ist eindentig eine $O C h \mathbf{C}^{\prime}$ bestimmt. Die Forderung lautet nun: Konvergiert $Q^{\prime}$ gegen $G^{\prime}$, so sollen die zugehörigen $\mathbf{C}^{\prime}$ einen Bogen bzw. eine Kurve als Limes $\left({ }^{18}\right)$ besitzen, der'(die) mit B nur endlich viele Pulkte gemeinsam hat. (Vgl. weiteres in $\mathrm{Nr}$. 3.1 2.).

A $\mathrm{nmerk} u \mathrm{ng}$. Man beachte, dass, falls keine Grundpunkte vorhanden sind (wie z. B. bei den Kreisen als $\mathrm{OCh}$ ), die Forderung (d) gegenstandslos wird und als von selbst erfüllt zn gelten hat.

Soweit im Folgenden von gemeinsamen Punkten zweier $O C h$ oder ciner $O C h$ mit dem Grundlogen die Rede ist und nicht ausdı ücklich anderes bemerkt wird, bleiben Grundpunkte stets ausser Betracht.

III. Jede OCh liegt normal zum Grundbogen: das soll heissen: Hat der Grundbogen $\mathbf{B}$ mit der OCh $\mathbf{K}$ die Punkte $P_{1}, \ldots, P_{r}$ gemeinsam $(r \geqq 3)$, wobei auch die Grundpunkte einbegriffen seien, so gibt es stets eine Anordnung der $P_{p}$, ftwa eben diese: $P_{1}, \ldots, P$, derart, dass bei einer der beiden Orientierungen von $\mathbf{B}$ bzw. $\mathbf{K}$ die $\boldsymbol{P}_{\rho}$ in eben dieser Reihenfolge durchlaufen werden; man nennt dann die Reihenfolge (die Anordnung) eine $\mathbf{n}$ atürliche auf $\mathbf{B}$ bzw. anf $\mathbf{K}$. Ist jede auf $\mathbf{B}$ natürliche Anordnung auch natürlich auf $\mathbf{K}$, so heissı $\mathbf{n} \mathbf{B}$ und $\mathbf{K}$ normal zueinander gelegen. (Aus dieser Definition folgt insbesondere, dass im Falle einer Grundkurve auch alle OCh Kurven sein müssen) ( $\left.{ }^{19}\right)$. Da wir im Folgenden stets $\mathrm{OCh}$ durch mindestens

(17) Betr. Schnitt- und Stuitzpunkt vgl. a. a. O. $\left({ }^{5}\right)$, Nr. 1.3. Soweit von Sehnittpunkten die Rede ist, wird hier der Durchsehnitt von $B$ mit jeder OCh als endlich angenommen,

(18) Ist das Grundgebiot $G$ kompakt, so sei die Konvergenz als metrische (im Haus. dorffschen Sinne) erklait, aber mit dem Zusatz betr. Teilbogen wie in Nr. 2.1., II., (c), (2'). Ist 6 nicht kompakt, so herrsche metrische Konvergenz in jedem kompakten Teil von $G$.

(19) Ist nämlich $\mathbf{B}$ eine Kurve, aber K£k ein Bogen mit $P_{i} \mathbf{B K}, i=1, \ldots, r$, wo $r \geq 3$, so sind \%. B. such $P_{2}, P_{3}, \ldots, P_{r}, P_{1}$ auf $\mathbf{B}$ natürlich angeordnet, aber nicht auf $\mathbf{K}$, falls $P_{1}, P_{2}, \ldots, P_{r}$ natürliche Anordnung auf $K$ ist. Da(Nach Vor., vgl. a.a.O. (15)) das Grund. gebiet von einer einfachen Kurve J begrenzt wird, kann man aber erforderlichenfalls die OCh, soweit sie Bogen sind, stets zu Kurven erweitern(durch Hin unahme eines geeigneten Teilbogens von J). 
$(k+1)$ Punkte von $\mathbf{B}$ betrachten, ist die vorstehende Definition einschlingig jedenfalls für $k>2$; für $k=1$ nur, wenn Grundpunkte vorhanden sind.

IV. Eindeutigkeit der k-Paratingenten an B. Wir bemerken zünächst: Es sei $Q$ ein Punkt von B. Ferner sei $V$ eine Umgebung ron $Q$ auf $\mathbf{B}$ und $\mathbf{K}$ eine OCh derart, dass $\mathbf{K} \mathbf{V}$ mindestens $Q$ von 'den Grundpunkten verschiedene Punkte enthält, wobei $q \geq \dot{k}$ ist. Zieht sich dann $Y$ auf den zugehörigen Punkt $Q$ zusammen und existiert dabei stets eine OCh der eben beschriebenen Art, und zwar bei festem $q$, so soll für $\mathbf{V} \rightarrow Q$ jeder Limes einer(konvergenten $\left({ }^{18}\right)$ Teil-)Schar solcher $0 \mathrm{Ch}$ als eine $\mathbf{k}-\mathrm{Par}$ atingente a $\mathbf{n}$ B in $Q$ bezeichnet werden. Die Vor. IV. besagt nun : Jede $\mathbf{k}-$ Paratingente ist ein durch $\mathrm{Q}$ eindeutig bestimmter einfacher Bogen (oder speziell eine einfache Kurve) $\mathrm{P}=\mathrm{P}(\mathrm{Q} ;$ q) der normal liegt $z u \mathbf{B}$ und mit $\mathbf{B}$ nur eine auf $\mathbf{B}$ nirgends dichte Menge von Punkten gemeinsam hat. Es soll ferner der gleiche Limes $P$ sich ergeben, wenn $q$, bei festgehaltenem $Q$ durch grössere Zahlen ersetzt wird, sofern zu diesen Zahlen zugehörige OCh $\mathbf{K}$ existieren.

Ist speziell $q \geq k+1$, so sprechen wir von $Q$ als von einem $k-S c h e i t e l$ von $B$ und dann von der zugehörigen Paratingente als einer k-Scheitel. paratingente. Für die k-Paratingente $\mathbf{P}$ im Punkte $Q$ bezeichnen wir $Q$ auch als Schmiegpunkt und die vom Schmiegpunkt verschiedenen Punkto aus BP als Begleitpunkte von $Q$ (auf BP) oder als (auf BP) mit $Q$ verbunden, unter Umständen (vgl. Nr. 3.1.1.) einschliesslich der Grı ndpunkte. Nach Voraussetzung liegen die mit $Q$ verbundenen Punkte nirgends dicht auf B. Die k-Paratingente ist (zufolge Vor.) stetige Funktion des Schmiegpunktes. Wir bemerken noch ausdrücklich, dass der chen bei dev Definition der Paratingente vernendete Punkt $Q$ auch Grundpunkt sein kann (wahrend die Punkte, durch welche die OCh $\mathbf{K}$ fostgelegt wird, verabre. dungsgemäss keine Grundpunkte sein dürfen).

Für den Fall, dass die OCh mit $\mathbf{B}$ nur endlich viele Punkte gemeinsam. haben, werde noch folgendes gefordert: Es sei $Q$ Schmiegpunkt der k-Paratingente $\mathbf{P}$ und Limes einer genau geraden bzw. ungeraden Anzahl von, keine Grandpunkt enthaltenden, Punkten von $\mathbf{B} \mathbf{K}^{\prime}$, wubei $\mathbf{K}^{\prime}$ die gegen $\mathbf{P}$ konvergierenden OCh sind; dann soll $Q$ auf $\mathbf{P}$ Schnitt-bzw. Stïlzpunkt mit B sein oder umgekehrt Stütz-bzw. Schnittpunkt, je nachdem $Q$ Grundpunkt ist oder nicht. Andererseits ist der Schmiegpunkt $S$ ron $\mathbf{P}$ Limes einer gera. den bzw. ungeraden Anzahl von Punkten aus BK', je nachdem $S$ Stiitzpunkt oder Schnittpunkt auf $\mathbf{P}$ mit $\mathbf{B}$ ist oder umgekehrt.

Etwas schwächer als Vor. IV. ist die (in IV. enthaltene) Vor.

IVa. Eindeutigkeit der k-Scheitelparatingenten; d. h. zu jedem k-Scheitel als Schmiegpunkt existiert genau eine k-Paratingente. Im übrigen soll jede k-Paratingente wieder ein einfacher, zu $B$ normal liegender Bogen (Kurve), braucht aber nicht durch jhren Schmiegpunkt 
eindentig bestimmt za sein (es sei denn, dass dieser Schmiegpunkt ein k-Scheitel ist).

Definition der Orientierung der Ordnungscharakteristiken und Paratingenten. Bei Festlegung einer Orientierung o von $B$ lassen sich die von uns zu betrachtende.1 OCh und k-Paratingenten folgendermassen orientieren: Durch o wird zunichst der Begriff der (positiven und negativen) Seite von $\mathbf{B}$ in der Umgebung eines jeden inneren Punktes von $\mathbf{B}$ festgelegt $\left({ }^{20}\right)$. Ferner wird duroh 0 eindeutig eine Orientierung anf einer jeden OCh $\mathbf{K}$ festgelegt, falls $K$ mit $\mathbf{B}$ mindestens 3 Punkte, etwa $R_{1}, R_{2}, R_{3}$ gemeinsam hat; ist nämlich $R_{1}, R_{2}, R_{3}$ die (bzw. falls $\mathbf{B}$ eine Kurve ist, eine) natïrliche Ascordnung der $R_{i}$ auf $\mathbf{B}$ bezüglich $\mathbf{0}$, so werde auf $\mathbf{K}$ diejenige Orientierung gewihlt, bei der $R_{1}, R_{2}, R_{3}$ auch auf $\mathbf{K}$ die(bzw. eine) natïrliche Anordnung ist. Von zwei Punkten auf $\mathbf{B}$ oder auf $\mathbf{K}$ (oder auf einem echten Teilbogen von I, bzw. $\mathbf{R}$, falls $\mathbf{L}$ bzw. $\mathbf{R}$ eine Kurve ist) wird dann als oberer bzw. unterer (als oberhalb bzw. unterhalb des anderen auf $\mathbf{B}$ oder $\mathbf{K}$ grolegen) derjenige bezeichnet, der dem anderen auf $\mathbf{B}$ oder $\mathbf{K}$ (beziiglich der Orientierung) vorangeht oder nachfolgt; dementsprechend ist die Bezeichnung obere und untere (einseitige) Umgebung des Punktes $Q$ auf $\mathbf{B}$ oder $\mathbf{K}$ zu verstehen. Sind $R, Q$ die Begrenzungspunkte eines orientierten Teilbogens ' $\mathbf{I}$ von $\mathbf{B}$ oder $\mathbf{K}$ (auf $\mathbf{B}$ oder $\mathbf{K}$ ), so heisse der obere bzw. unter von ihnen End-bzw. A nfangspunkt von T. Beim Grenzïbergang zu einer Paratingente liasst sich die Orientierung von den OCh auf die Paratingente ubertragen. Die für uns spaterhin in Betracht kommenden OCh $\mathbf{K}$ und $\mathbf{k}$-Paratingenten $\mathbf{P}$ besitzen woiter die Eigenschaft, dass eine untere Umgebung auf $\mathbf{K}$ bzw. auf $\mathbf{P}$ eines ansgezeichneten Punktes von BK bzw. des jeweiligen Schmieg* punktes von $\mathbf{P}$ fremd ist zur einen Seite von $\mathbf{B}$; wir bezeichnen dann zwei OCh $\mathbf{K}^{\prime}, \mathbf{K}^{\prime \prime}$ bzw. zwei $\mathbf{k}$-Paratingenten $\mathbf{P}^{\prime}, \mathbf{P}^{\prime \prime}$ als gleichaxtig bzw. als ungleichartig (grelegen bezüglich $\mathbf{B}$ ), je nachdem untere Umgebungen der ausgezeichneten Punkte von $\mathbf{K}^{\prime}, \mathbf{K}^{\prime \prime}$ bzw. der Schmiegpunkte von $\mathbf{P}^{\prime}, \mathbf{P}^{\prime \prime}$ fremd sind zur gleichen Seite bzw. zu verschiedenen Seiten von B. Auch die Schmiegpunkte werden entsprechend als gleich-bzw. ungleichartig bezeichnet, insbesondere spricht man von gleich-bzw. ungleichartigen k-Scheiteln.

$\mathrm{Z}$ us atz. Ist $A$ ein, von den Grundpunkteli verschiedener Punkt des Grundbogens, so ist das System aller, den Punkt $A$ enthaltender .OCh aus $k$ ein System $\mathbf{k}^{*}$ von OCh mit der Grundzahl $k^{*}=k-1$. Die $\mathbf{k}^{*}-\mathrm{Sch}$ eitel sind dann Erstens diejenigen, von $A$ verschiedenen Punkte von $\mathbf{B}$, deren $\mathbf{k}-$ Para.

(20) Ist 1 eine Kurve, so entsprechen die beiden "Seiten" von $\mathbf{B}$ etwa den von $\mathbf{B}$ begrenzlen beschrinkten und nicht beschrinkkien Gebiet (gemäss des Jordanschen Kurven-

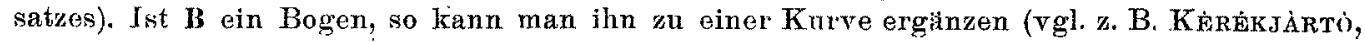
B. v., Vorles. über Topologie I., Berlin 1923, s. 69). 
tingenten den Punkt $A$ enthalten; Zweitens der Punkt $A$ selbst, wenn $A$ ein $k$-Scheitel ist.

2.1.1. A nmerkung. Der Paratingentenbegriff und die an ihn gestellten Forderangen werden bei der Formulierung von Vor. IV. nur im Interesse der Anschaulichkeit herangezogen. In Wirklichkeit ist der für unsere Zwecke wesentliche Inhalt der Vor. IV. davon unabhăngig. Es genügt nämlich, folgendes vorauszasetze... Die sämtlichen, in $\mathbf{B K}$ enthaltenen Pankte seien $X_{1}, \ldots, \mathrm{X}_{q}$; ferner sei $1 \leqq t \leqq q$ und $q_{1}+\ldots+q_{t} \geq k$ mit $q_{\tau}>0,1 \leqq \tau \leqq t$; ausserdem sollen $t$ verschiedene Punkte $Q_{1}, \ldots, Q_{t}$ anf $\mathbf{B}$ existieren derart, dass unter den $X_{1}, \ldots, X_{q}$ insgesamt $p_{\tau}^{\prime}$ gegen $Q_{\tau}$ konvergieren, $\tau=1, \ldots, t$, wobei $0 \leq q_{\tau}^{\prime} \leq q_{\tau}$, und $k_{1}^{\prime}+\ldots+k_{t}^{\prime}=k$. Dann konvergieren die übrigen Punkte $X_{\tau}$, soweit sie beim Grenzübergang nicht verloren gehen, gegen feste, nur durch die $Q_{\tau}, q_{\tau}^{\prime}$ bestimmte Punkte von $\mathbf{B}$, die teilweise oder sämtlich untereinander und mit den $Q_{\tau}$ zusammenfallen können; und für $t=1$, also $q_{\mathrm{i}}^{\prime}=q$ liegen die beim Grenzübergang auftretenden OCh $K$ sämtlich gleichartig, wenn der unterste ron den gegen $Q_{1}$ konvergierenden Punkten unter den $X_{1}, \ldots$ von unten her gegen $Q_{1}$ konvergiert und ausgezeichnet wird (vgl. Nr. 3.1.) Man kann auch noch weitergehen und entsprechend die Benutzung des Begriffes der OCh zu vermeiden, also sämtliche Aussagen lediglich mit Hilfe des Begriffes des $q$-tupels von Punkten auf $\mathbf{B}$ zu formulieren suchen.

\subsection{Formulierung der verallgemeinerten Sätze.}

Wir bezeichnen als k-Ordnungswert, karz k-OW, des Grundbogens B, und allgemeiner eines beliebigen Teilbogens ' $\mathbf{l}$ von $\mathbf{B}$, die grösste Müchtigkeit $r \geq k$, welche der Durchschnitt von $\mathbf{T}$ mit einer OCh $\mathbf{K}$ besitzt (falls dieses Maximum $r$ existiert); Grundpunkte werden bei der Bestimmung der Mäch. tigkeit von TK nicht mitgezahlt. Von endlichem bzw. beschräktem k-OW sprechen wir, wenn die Mächtigkeit von BK für alle OCh $\mathbf{K}$ endlich ist bzw. eine endliche obere Schranke besitzt. Ferner bezeichnen wir einen Punkt PEB als vom k-Ordnungswert $p \geq k$, wenn jede hinreichend kleine Umgebung von $P$ auf $B$ den k-Ordnungswert $p$ besitzt. Die Punkte, deren $\mathbf{k - O W}$ mindestens gleich $(k+1)$ ist, sind identisch mit den früher als k-Scheitel bezeichneten Punkten von B. Dabei kann ein k-Scheitel $\mathbb{S}$ zugleich Grundpunkt sein (vgl. Nr. 2.1, IV.); in diesem Falle gibt es zur k-Scheitelparatingente in $S$ beliebig benachbarte OCh, welche ausser $S$ noch mindestens $(k+1)$ verschiedene zu $S$ beliebig benachbarte Punkte mit B gemeinsam haben.

Die in Aussicht genommenen Verallgemeinerungen der in Nr. 1.1. bis 1.3. angegebenen Sătze sowie einige damit zusammenhängende Bemerkungen können nun so ausgesprochen werden:

(A) Es seien die Voraussetzungen I. - III. (Nr. 2.1.) erfüllt:

(1) Besitat der Grundbogen B mindestens den k-Ordnungswert $(\mathrm{kt}+1)$, 
wobei $\mathrm{k} \geq 1, \mathrm{t} \geq 1$, so ist die Anzahl der $\mathrm{k}-$ Scheitel von $\mathbf{B}$ mindestens $\mathrm{t}$ und, wenn B eine Kurve ist, mindestens $(t+1)$. (Verallgemeinerter Zwei. scheitelsatz).

(2) Jeder, nur isolierte ('11) k-Scheitel enthaltende, Grundbogen B ist Summe von, bis auf ihve Begrenzungspunlte fremden (abgeschlossenen) Bogen je vom $\mathrm{k}-$ Ordnungswert $\mathrm{k}$ derart, dass die. im Innern von $\mathbf{B}$ gelegenen Begrenzungspunkte dieser Bogen genau die $\mathbf{k}-$ Scheitel von $\mathbf{B}$ sind. (Begrenzungspunkte des Grundbogens sind nur dann k-Scheitel, wenn ihr k-Ordnungswert grösser als $(k+1)$ und der Begrenzungspunkt daher kein isolierter k-Scheitel ist).

(B) Es seien die Voraussetzungen I. - IV bzw. IVa. (Nr. 2.1.) erfüllt:

(1) Besitat der Grundbogen B mindestens den $\mathrm{k}-O r d n u n g s w e r t ~ \mathrm{p} \geq \mathrm{k}+1$, so ist die Anzahl der $\mathrm{k}-$ Scheitel von $\mathbf{B}$ mindestens $(\mathrm{p}-\mathrm{k})$. Ist $\mathrm{C}$ eine Grundkurve ${\left({ }^{2 \cdot}\right)}^{-1}$ von mindestens dem $\mathrm{k}$-Ordnungswert $\mathrm{p} \geq \mathrm{k}+1$, so ist die Anzahl der k-Scheitel von $\mathrm{C}$ mindestens gleich $\mathrm{p}$.

(2) Besitzt der Grundbogen $\mathbf{B}$ höchstens den k-Ordnungswert ( $\mathrm{k}+1)$, so ist die Anzahl der $\mathbf{k}-$ Scheitel von $\mathbf{B}$ höchstens gleich $(\mathrm{k}+1)$.

(3) Die Grundkurve (":) c besitat genau den $\mathbf{k}$-Ordnungswert $(\mathrm{k}+1)$ dann und nur dann, wenn die Anzahl der k-Scheitel von $\mathbf{C}$ genau gleich $(\mathrm{k}+1)$ ist $\left.{ }^{23}\right)$.

(4) Jeder is olierte $\mathrm{k}-\mathrm{S}$ cheitel besitat genau den $\mathrm{k}-$ Ordnungsvert $(\mathrm{k}+1)$. Jeder genau ein en k-Scheitel enthaltende Grundbogen besitat genau den k-Ordnungswert $(\mathrm{k}+1)$.

(5) Je zwei, auf $\mathbf{B}$ unmittelbar benachbarte (d.h. durch keinen $\mathbf{k - S c h e i t e l}$ auf $\mathbf{B}$ getrennte) k-Scheilel sind gleich-oder ungleichartig, je nachdem sie auf B durch eine ungerade oder gerade Anzahl von Grundpunkten von einander getrennt werden. Fallt dabei ein $\mathrm{k}-$ Scheitel $\mathrm{S}$ in einen Grundpunkt $\mathrm{G}$, so ist $\mathrm{G}$ als ein im Sinne der Orientierung von B auf $\mathrm{S}$ folgender (oberhalb $\mathrm{S}$ liegender) Grundpunkt zu rechnen (ganz entsprechend der auf die Orientierung von B Bezug nehmenden Definition der Gleich-bzw. Ungleichartigkeit

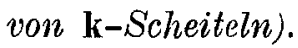

Zusat\%. Es sei hervorgehoben, dass zum Beweise der Sätze (B) (2) und (B) (4) nur die Vor. IVa. (nicht IV.) benötigt wird. Die beim Beweise der Sätze (B) (1) und (5) herangezogene Vor. IV. liesse sich ubrigens auch noch abschwächen, wie wir später zu zeigen hoffen.

(21) Ein k-Scheitel $S$ heisst is oliert, wenn in einer Umgebung von $S$ auf $\mathbf{B}$ ausser $S$ kein k-Scheitel existiert. - Zur Feststellung in (B) (4), dass unter unseren Vorausset. zungen I. - IVa. jeder isolierte k-Scheitel genau den k-OW $(k+1)$ besitzt, vgl. Entspre* chendes für Raumkurven bei J. Sauter, Math. Zeitschr, 42 (1937), 592, Zusatz.

(22) Die OCh sind dann auch sümtlich Kurven. Vgl. Nr. 2.1., III.

(23) Falls die eindentige Existenz der k-Paratingente nicht voransgesetzt wird, gilt der Satz im allgemeinen nicht. $\nabla$ gl. das Beispiel in Nr. 3.2.1.2. 
Die Sütze (A) werden in Nr. 3.2. bewiesen, die Siatze (B) (4) bzw. (1) bzw. (2) bzw. (5) in Nr. 3.3. bzw. Nr. 3.4. bzw. Nr. 3.5. bzw. Nr. 3.6. Der Satz (B) (3) ist unmittelbare Folge aus den Sătzen (B) (1) und (2).

\section{Beweise.}

3.1. Folgerungen aus den Voraussetzungen I-II. in Nr. 2.1. (ohne Vor. I Va.) 3.1.1. Irgend $r \geq 1$ in natürlicher Anordnung auf $B$ befindliche Schnittpunlte $\left({ }^{19}\right) S_{1}, \ldots, S_{2}$ von $\mathbf{B}$ mit einer OCh $\mathbf{K}$ bezeichnen wir als ein $r$-tupel $\gamma$; dabei werden Grundpunkte als Punkte eines solchen $r$-tupels nicht mitgezühlt. Die Ordnungscharakteristik K heisse zum $r$-tupel $\gamma$ gehörig. Der (bei der zu Grunde gelegten und dann stets festgehaltienen Orientierung von B) unterste Punkt $S_{t}$ von $\gamma$ heisse auch der Anfangspunkt von $\gamma$. Der alle $S_{\phi}$ enthaltende Teilbogen $G$ von $B$ mit $S_{1}$ als Anfangs-und $S_{r}$, als Endpunkt werde als zu $\gamma$ gehöriger (Teil-) Bogen bezeichnet. Sind in $\gamma$ ausser den $S_{\rho}, \rho=1, \ldots, r$, (und etwaigen Grundpunkten) keine weiteren Schnittpunkte aus BK enthalten, so heisse $\gamma$ un unterbuchen. Enthalt BK ausser den $S_{\beta}$ (und ausser Grundpunkten) noch andere Punkte, so heissen diese mit $\gamma$ oder mit den: $S_{p}$ verbunden (falls $r \geq k$ ). Ein Grundpunkt $G$ soll als mit einem $r$-tupel $\gamma$ verbunden gelten, falls $G \mathrm{Schnittpunkt} \mathrm{ist}$ von $\mathbf{B}$ mit der, $\gamma$ tragenden $\mathrm{OCh}$; soweit es nicht ausdrücklich hervorgehoben wird, sind aber unter "mit $\gamma$ verbundenen Punkten nur von Grundpunkten verschiedene Punkte zu verstehen. Es sei $\gamma^{\prime}$ bzw. $\gamma^{\prime \prime}$ ein $r^{\prime}$-bzw. $r^{\prime \prime}$-tupel $\left(r^{\prime} \geq 1, r^{\prime \prime} \geq 1\right)$ mit dem Anfangspunkt $S^{\prime}$ bzw. $S^{\prime \prime}$ und mit $\mathbf{K}^{\prime}$ bzw. K' als zugehöriger OCh. Sind dann untere Umgebungen von $S^{\prime}$ auf $K^{\prime}$ und von $S^{\prime \prime}$ auf $\mathbf{K}^{\prime \prime}$ fremd zur gleichen bzw. zu verschiedenen Seiten von $\mathbf{B}$, so sollen $\gamma^{\prime}$ und $\gamma^{\prime \prime}$ sowie $\mathbf{K}^{\prime}$ und $\mathbf{K}^{\prime \prime}$ als gleich-bzw. als ungleichartig (gelegen) zu B für $S^{\prime}, S^{\prime \prime}$ bezeichnet werden. Wir bemerken noch: Reduktionssatz $\left({ }^{24}\right)$ : Ist $\mathbf{B}$ von endlichein $\mathbf{k}-\mathrm{OW}$, d.h. hat $\mathbf{B}$ mit jeder $\mathrm{OCh}$ nur endlich viele Punkte gemeinsam, sind ferner $s$ Punkte $P_{1}, \ldots, P_{s}$ in $\mathbf{B K}$ enthalten, so existieren in beliebiger Nachbarschaft von $\mathbf{K}$ solche $O C h . \mathbf{K}^{\prime}$, die zu einem $s$-tupel $\gamma^{\prime}$ gehören, das im Innern von $\mathbf{B}$ liegt und dessen Punkte beliebig benachbart sind je zu einem der $P_{1}, \ldots, P_{s}$; dabei kann überdies gefordert werden, dass BK' nur Schnittpunkte enthalt.

3.1.2. Spezieller M on otoniesatz $\left({ }^{25}\right)$. Der Grundbogen B sei wieder von endlichem k-OW (d.h. es onthalte BK nur éndlich viele Puinkte für jede OCh $K$ ). Enthält BK nicht lauter Schnittpunkte, so gibt es za K beliebig

(24) Vgl. a. a. 9. (5), Nr. 2.4. Ver Reduktionssat\% gilt anch dann, wenn Grundpunkte auf $B$ liegen.

(25) Vgl. a. a. O. (5), Nr. 3.2. Der Monotoniesatz gilt auch, wonn Grundpunkte aut B liegen. 
benachbarte OCh $\mathbf{K}^{\prime}$, sodass $\mathbf{B K}^{\prime}$ nur Schnittpunkte enthält und zwar min. destens ebensoviele, als Punkte in BK vorhanden sind $\left({ }^{26}\right)$. Es habe nun $K$ mit 13 nur Schnitpunkte gemeinsam, etwa $S_{\mathfrak{i}}, \ldots, S_{n}$, und zwar mindestens $(k+1)$. Werden dann irgend $(k-1)$ nnter diesen $S_{p}$ festgehalten und wind einer der übrigen durch eine hinreichend kleine Abänderung von $\mathbf{K}$ monoton und stetig aus seiner Ausgangslage heraus «bewegt», so «bevegen» sich je zwei der ibrigen Punkte unter den $S_{g}$ (streng monoton) im gleichen oder entgegengesetzten Sinne auf $\mathbf{B}$, je nachdem zwischen ihnen eine ungtrade oder gerade Anzahl beveglicher Schnittpunkte liegen.

Ritckt im Laufe dieser Bewegung ein berveglicher Punkt $\mathrm{S}$ mit einem Grundpunkt $\mathrm{G}$ zusammen, aber nicht gleichzeitig mit einem ander $n$ beweglichen, ihm entgegenruckenden Punkt und werden nicht gleichzeitig Punkte auf BK gewonnen, so wird $S$ den Pnnkt $G$ unter Beibehaltung seiner Bewegungsrichtung überschreiten, falls die ubrigen beweglichen Punkte ihre Bewegungsrichtung nicht indern. (Ansser Betracht bleiben kann hier der Fall, dass ein beweglichér Punkt $S$ mit einem festgehaltenen Punkt zusammenrickt, der nicht Grund. punkt ist, weil sich das durch passenden Wechsel der festgehaltenen und der benachbarten beweglichen Punkte jeweils umgehen lasst). Jje Bewegung von $S$ verlihuft mithin so, als ob der Grundpunkt $G$ gar nicht vorhanden wire: bemerkbar macht sich der Durchgang von $S$ durch $G$ nur insofern, als sich beim Durchgang die Lage der unteren Umgebung von. $S$ auf $\mathbf{K}$ gegenüber $B$ indert. (Der Beweis dieser Beh, ergibt sich aus Vor. II. (d) in Nr. 2.2.). Weiter folgt: Überschreitet bei monotoner Änderung (und $(k-1)$ festen Punkten) der Anfangspunkt $S$ des betrachteten $r$-tupels $\gamma$ einen Grundpunkt $F$, ohne mit einem (anderen) beweglichen Punkt zusammenzurticken, so liegt nach dem Jurchgang von $S$ durch $F$ ein zum ursprünglichen ungleichartiges $r$-tupel vor (vorausgesetzt, dass keine Punkte von $\gamma$ «verloren» gingen); wir sagen dafür kurz anch: $\gamma$ geht in ein $z u \quad \gamma$ ungleichartiges r-tupel über, falls der Anfangspunkt $\mathrm{S}$ von $\gamma$ einen Grundpunkt überschreitet, sonst in ein gleichartiges.

Hinsichtlich der im Verlaufe einer monotonen Änderung eines $r$-tupels mit $(k-1)$ festgehaltenen Punkten möglichen "Gewinne» und «Verluste» sei noch folgendes bemerkt: "Neue Punkte" können nur auftreten'|"gewonnen werden》| innerhalb "abnehmender» Teilbogen, d.h. solcher Teilbogen, die von zwei beweglichen, auf $\mathbf{B}$ sich einander nähernden Punkten des $r$-tupels begrenzt werden. Demgegenüber treten in einem «w a chsenden» ("zunehmenden»). d.h. von zwei auf $B$ sich voneinander entfernenden beweglichen Punkten begrenzten, Teilbogen Gewinne höchstens dann auf, wenn der Teilbogen abnehmende Teilbogen enthalt, also keine Gewinne, wenn er

(29) Vgl, a. a. O. (4). Betr. die Begriffe "Gewinn" und "Vmlust* rgl. z. B. a. a. O. (14). 2. Abh., Nr. 1.5.1. fr. 
im Innern fremd ist zur OCH, die zum $r$-tupel gehört. Dagegen sind VerInste, hervorgerufen durch das Zusammenruicken zweier beweglicher Punkte, sowohl in abnehmenden als in zunehmenden Teilbogen möglich.

3.1.3. Kontraktionssatz $\left.{ }^{27}\right)$. Es sei $B$ wieder von endlichem k-OW. In einem $(k+1)$-tupel $\gamma=\left(S_{1}, \ldots, S_{k+1}\right)$ kann man unter Festhalten von $(k-1)$ der $S_{x}$ die übrigen beiden Schnittpunkte dem Monotoniesatze gemäss so bewegen, dass der zu $\gamma$ gehörige Teilbogen 'W' von B abnimmt, ferner dass die beweglichen Punkte uber keinen der jeweils festgehaltenen Punkte von $\gamma$ hinwegrücken, sondern höchstens über Grundpunkte, und auch dass keine beweglichen Punkte znsammenrücken. Sollte eines der hiermit ausgeschlos. senen Vorkommnisse eintreten, so werde vorher die Bewegung unterbrochen und der Prozess bei anderer Wahl der festen Punkte so fortgesetzt, dass $\mathbf{T}$ auch weiterhin abnimmt. Dabei kann o.B.d.A. $\gamma$ als ununterbrochen angenommen werden, weil andernfalls (z.B. auch bei Gewinnen innerhalb T) aus $\gamma$ (ev. durch Übergang zu einer beliebig benachbarten $O C h$ ) ein mit $\gamma$ gleichar. tiges ununterbrochenes, in $\mathbf{T}$ enthaltenes neues $(k+1)$-tupel erhalten werden kann (vgl. Nr. 3.1.1., Reduktionssatz). Man bezeichnet eine solche monotone Änderung von $\gamma$ (mit Verkleinerung von $\mathbf{T}$ ) als eine Kontraktion von $\gamma$. Werden mehrere solche Kontraktionen (mit wechselnden festen und beweglichen Punkten hintereinander ausgeführt gemischt mit beliebig klein wählbaren Änderungen der OCh (denen keine monotone Änderung des $(k+1)$-tupels zu entsprechen braucht, vgl. die Anwendung des Reduktionssatzes in Nr. 3.1.2., Anfang, sowie oben in Nr. 3.1.3.), si) spricht man von einem Kontrak. tionsprozess $\left({ }^{28}\right)$. Der Kontuaktionssatz( (27) besagt dann: Zu jedem beliebig vorgegebenen $(k+1)$-tupel $\gamma$ existiert ein Kontraktionsprozess, vermöge dessen sich $\gamma$ auf einen inneren Punkt $Q$ des zu $\gamma$ gehörigen Teilbogens T zusammenziebt (Dabei kann $Q$ auch ein Grundpunkt sein). Es liegt $Q$ ober. halb der Anfangspunkte aller beim Prozess auftretenden $(\mathrm{k}+1)$-lupel.

Dieser Kontraktionssalz gestattet (unter den Vor. I..III.) nachsiehende

Verschärfung: Der zu einem $(k+1)$-tupel gehörige Kontraktionsprozeșs kann sttets folgendermassen eingerichtet werden: Die bei den einzelnen Schritten des Kontraktionsprozesses auftretenden $(k+1)$-tupel $\gamma^{\prime}$ sowie die den $\gamma^{\prime}$ zugehörigen $0 \mathrm{Ch} \mathbf{K}^{\prime}$ liegen gleich-bzw. ungleichartig, je nachdem der Anfangspunkt des ursprünglichen $(k+1)$-tupels $\gamma$ beim Übergang zum Anfangspunkt von $\gamma^{\prime}$ eine gerade bzw. ungerade Anzahl Grundpunkte (in Richtung nach oben auf $\mathbf{B}$ ) uberschritten hat. Die $\mathbf{K}^{\prime}$ (und $\gamma^{\prime}$ ) sind schliesslich alle gleichartig untereinander.

(2r) Vgl. für das Folgende a. a. 0. (5), Nr. 4.2.; fernel a. a. O. ( $\left.{ }^{2}\right)$, Nr. 2.4. ff. Der Kontraktionssatz gilt unter den Vor. 1. - II1, auch, wenn Grundpunkte auf B liegen.

$\left.{ }^{28}\right) \mathrm{V}$ gl. a. a. O. (12), N1.2.3.1. 
Zusatz. Falls Nr. 2.1., Vor. IVa., hinzugenommen wird, gilt. uberdies: Die ' $K$ ' und $\gamma^{\prime}$ sind schliesslich alle gleichartig zu der k-Scheitelparatingente in $Q$ an $\mathbf{B}$ (gegen welche die $\mathbf{K}^{\prime}$ konvergieren).

Bew. Bei dem frtiher $\left({ }^{29}\right)$ gegebenen Beweise des Kontraktionssatzes wurde die eben formulierte Verschärfung noch nicht berucksichtigt; man erhalt sie aber durch folgende Modifikation: Es sei $\gamma_{1}=\left(S_{1}, \ldots, S_{k+1}\right)$ das ursprïnglich vorliegende $(k+1)$-tupel und $\mathbf{K}_{1}$ die zugehörige $O C h$; wenn nötig erzwingen wir durch eine beliebig kleine Abänderung von $\mathbf{K}_{1}$ in $\mathbf{K}_{1}^{\prime}$, dass BK, nur Schnittpunkte enthält und dass $K_{1}^{\prime \prime}$ zu einem mit $\gamma_{1}$ gleichartigen $(k+1)$-tupel $\gamma_{i}^{\prime}$ gehört, wobei $\gamma_{i}^{\prime}$ ununterbrochen ist und wobei der Anfangs. punkt von $\gamma_{1}^{\prime}$ nicht unterhalb des Anfangspunktes von $\gamma_{1}$ liegt (gemäss Nr. 3.1.1., Reduktionssatz). Indem wir nun definitionsgemäss die $S_{1}$ in $\gamma_{1}$ als in naturlicher Anordnung (auf $\mathbf{B}$ und $\mathbf{K}$ ) voraussetzen, balten wir $S_{3}, \ldots, S_{k+1}$ fest und kontrahieren $S_{1}, S_{2}$. Es ist dann ' $S_{1}$ End-und $S_{2}$ Anfangspunkt je eines wachsenden Teilbogens $\mathbf{T}_{1}$ bzw. $\mathbf{T}_{2}$ von $\mathbf{B}$ sodass weder in $\mathbf{T}_{1}$ noch in $\mathbf{T}_{2}$ Gewinne stattfinden können. Entweder kann man nun $S_{1}$ und $S_{2}$ einander beliebig nahe bringen, wobei das entstehende $(k+1)$-tupel $\gamma_{2}$ gleichartig oder ungleichartig ist mit $\gamma_{1}$; je nachdem $S_{1}$ beim Übergang von $\gamma_{1}$ zu $\gamma_{2}$ eine gerade oder ungerade Anzahl Grundpunkte uberschritten hat. Oder es werden auf dem abnehmenden Teilbogen, der $S_{t}$ als Anfangs-und $S_{\Omega}$ als Endpunkt besitzt, endlich viele Paare von Schnittpunkten gewonnen; dann ersetzen wir $S_{2}$ durch den am nachsten oberhalb $S_{1}$ gelegenen nenen Schnittpunkt $S_{2}$, ferner $\gamma$ durch ein ununterbrochenes $(k+1)$-tupel $S_{1}{ }^{\prime} S_{2}{ }^{\prime} S_{3}{ }^{\prime} \ldots S_{k+1}^{\prime}$ und setzen die Kontraktion bei festen $S_{3}{ }^{\prime}, \ldots, S_{k+1+1}^{\prime}$ fort. Durch Fortsetzung dieses Verfahrens und ev. vollstandige Induktion im Kontinuum $\left({ }^{30}\right)$ ergibt sich schliesslich ein $(k+1)$-tupel $\gamma^{\prime}$ mit $\mathbf{K}^{\prime}$ als zugehöriger OCh derart, dass in $\mathbf{B K}^{\prime}$ ein ununter. brochenes $(k+1)$-tupel $\gamma_{1}^{\prime \prime}=\left(S_{1}^{\prime \prime}, \ldots, S^{\prime \prime}{ }_{k+1}\right)$ mit dem gleichen Anfangspunkt $S_{1}^{\prime \prime}$ wie $\gamma^{\prime}$ enthalten ist von folgender Beschaffenheit:

(A) Es liegt $S_{1}^{\prime \prime}$ oberhalb $S_{1}$ und ist Endpunkt eines, $\mathbf{T}_{1}$ enthaltenden, zu BK' fremden (offenen) Teilbogens von $\mathbf{B}$;

(B) Es liegt $S^{\prime \prime}{ }_{k+1}$ nicht oberhalb $S_{k+1}$;

(C) Es liegt $\gamma_{1}^{\prime \prime}$ gleichartig bzw. ungleichartig mit $\gamma_{1}$, je nachdem zwischen $S_{1}$ und $S_{1}^{\prime \prime}$ eine gerade oder ungerade Anzahl Grundpunkte liegt;

(D) Die Schnittpunkte $S_{f}^{\prime \prime}, \ldots, S_{R}^{\prime \prime}$ für ein gewisses $R \geq 2$ besitzen beliebig kleine Abstände voneinander.

Dass hierbei (A), (B) und (C) erfullbar sind, ist unmittelbar einzusehen; ebenso dass (D) für $R=2$ gilt. $\mathrm{Zu}$ Beweise des verschärten Kontraktions.

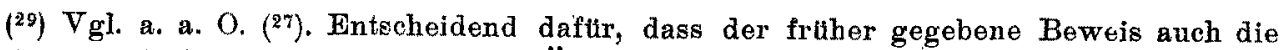
Verschärfung liefert, ist, dass bei den Überlegungen a. a. $\mathrm{O}$. (5), Nr. $4.7_{1}$. und $4.7_{2}$, nur Kontraktionsprozesse verwendet werden, vermittelst deren lauter $(k+1)$-1upel mit den Eigenschaften $(A)-(D)$ (vgl. oben im Text) gewonnen werden.

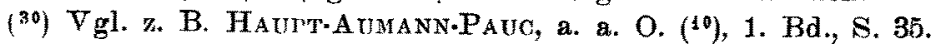


satzes ist daher noch zu zeigen, dass $R=k+1$; des Beweis hierfür verläuft im wesentlichen wie früher $\left(^{(2 !}\right)$.

Betr. Bew. des Zusatzes. Im Falle die Vor. IVa. im Nr. 2.1. erfült ist, gilt folgendes: Ziehen sich die beim Kontraktionsprozess auftretenden $(k+1)$ tupel $\gamma$ auf den Punkt $Q$ zusammen, wobei also die Anfangspunkte der $\gamma$ monoton nach oben gegen $Q$ sich bewegen, so existiert eine, nur durch das ursprungliche $(k+1)$-tupel bestimmte untere Umgebung $\mathbf{V}$ von $Q$ auf $\mathbf{B}$ mit der folgenden Eigenschaft: $Z \mathbf{~} \mathbf{V}$ gehört eine untere Umgebung $\mathbf{U}$ von $Q$ auf

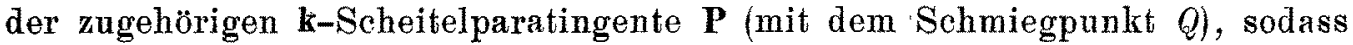
$\mathbf{U}$ mit $\mathbf{V}$ keine Schnittpunkte gemeinsam hat, also $\mathbf{U}$ in einer geeigneten zweidimensionalen Umgebung von $Q$ ganz auf der einen Seite von $\mathbf{B}$ verläuft. Und das Gleiche gilt für eine untere Umgebung des Anfangspunktes von $\gamma$ auf der zu $\gamma$ gehörigen OCh für schliesslich alle $\gamma$. Der Beweis folgt daraus, dass vermöge (A) und. (C) die Beh. in der Tat für schliesslich alle $\gamma$ erfüllt ist, weil nämlich zwischen $Q$ und schliesslich allen $S_{1}$ " keine Grundpunkte mehr liegen; und da die $\mathbf{k}-$ Scheitelparatingente $\mathbf{P}$ eindeutig bestimmt ist (Vor. IVa.) liegen $(k+1)$-tupel aus verschiedenen, zu $\mathbf{P}$ führenden Kontraktionsprozessen stets gleichartig, sofern sie zu $Q$ hinreichend benachbart sind.

3.2. Beweis der Sätze (A) (1) und (2) in $\mathrm{Nr}$. 2.2. .

Diese Beweise ergeben sich aus den folgenden Sutzen, denen lediglich die Vor. I.-III. (Nr. 2.1.) zu Grunde liegen.

3.2.1. Sind $\mathrm{S}_{4}, \ldots, \mathrm{S}_{\mathrm{k}+1}$ (von den Grundpunkten verschiedene) Schnittpunkte des Grundbogens $\mathbf{B}$ mit der Ordnungscharakteristik $\mathbf{K}$ in natïrlicher Anordnung $(\mathrm{k} \geq 1)$, so enthäll der zum $(\mathrm{k}+1)$-tupel $\gamma=\left(\mathrm{S}_{1}, \ldots, \mathrm{S}_{\mathrm{k}+1}\right)$ gehörige (abgeschlossene) Teilbogen $\mathbf{T}$ von $\mathbf{B}$ (mit $\mathrm{S}_{1}$ bzw. $\mathrm{S}_{\mathrm{k}+1}$ als Anfangs-bzn. als Endpunkt) in seinem Inneren mindestens einen $\mathbf{k}$-Scheitel (der definitionsgemäss auch mit einem Grundpunkte zusammenfallen kamn). (Ohne Vor. I Va. (Nr. 2.1.)).

Bew. Hat ein, ganz im Innern von $\mathbf{T}$ gelegener, abgeschlossener Teilbogen $\mathbf{T}^{\prime}$ von $\mathbf{T}$ mit mindestens einer $0 \mathrm{Ch} \mathbf{K}^{\prime}$ unendlich viele Punkte gemeinsam, so besitzt die Menge ' $/ \mathbf{K}$ ' wegen der Kompaktheit von $\mathbf{T}$ mindestens einen Häufungspunkt in 'T' also im Innern von 'T; jeder solche Haufungspunkt ist aber ein $\mathbf{k}-$ Scheitel, weil jetzt $\mathbf{K}^{\prime}$ selhst eine $\mathbf{k}$-Scheitelparatingente ist. Besitzt hingegen jedtr solche Teilbogen ' $T$ ' von $\mathbf{T}$ endlichen $\mathbf{k}-0 \mathrm{~W}$, so gehe man vermittelst ein $\mathrm{r}$ geeigneten, belirbig kleinen Kontraktion von $\gamma$ zu einem $(k+1)$-tupel $\gamma^{\prime}$ uber, das im Innern von $\mathbf{T}$, also in einem Teilbogen ' $\mathbf{T}^{\prime}$ enthalt $\mathrm{n}$ ist; da $\mathbf{T}^{\prime}$ nach Annahme endlichen $\mathbf{k}-0 \mathrm{~W}$ besitzt, ist der Kontraktionssatz auf $\gamma^{\prime}$ anwendbar, woraus die Beh. folgt.

3.2.1.1. Beweis von Satz (A) (1) (Nr. 2.2.).

Verallgemeinerter Zweischeitelsatz. Vor. Es sei B ein Grundbogen, velcher nur den Vor. I.-III. von Nr. 1.2., aber nicht der Vor. IVa., zu 
genügen braucht. Ferncr soli $\mathbf{B}$ mindestens den $\mathbf{k}-\mathrm{OW}(\mathrm{kt}+1)$ besitzen $(\mathrm{k} \geq 1$, $\mathrm{t} \geq 1$ 1). Der Fall, dass der k-OW von B nicht endlich ist, sei einbegriffen.

Beh. Die Anzahl der in Innern von $\mathbf{B}$ gelegenen $\mathbf{k - S c h e i t e l}$ ist mindestens gleich $\mathrm{t}$ und, falls $\mathrm{B}$ eine Grundkurve ist, mindestens gleich $(\mathrm{t}+1)$.

Bew. Indirekt. Es sei alse die Anzahl der k-Scheitel $Q_{i}$ innerhalb B gleich $s \leq t-1 \mathrm{bzw}$. (falls B eine Kurve ist) gleich $s \leq t$. Dann ist B Summe von $(s+1)$ oder, falls $\mathbf{B}$ eine Kurve ist, von $s$ Teilbogen $\mathbf{T}_{j}$, die abgeschlossen in $B$ sind, parweise keine inneren Punkte gemeinsam haben und unter deren Endpunkten alle k-Scheitel $Q_{i}$ enthalten sind. Es sind dann nur folgende Falle denkbar: Entweder besitzen alle $\mathbf{T}$, endlichen $\mathrm{k}-\mathrm{OW}$, dann auch $\mathbf{B}$ selbst, sodass dor Reduktionssatz anwendbar ist und o.B.d.A. voransgesetzt werden kann. dass alle Punkte von BK Schnittpunkte sind $\mathfrak{w}_{i}$ d dass ihre Anzahl für passende $\mathbf{K}$ mindestens gleich $(k t+1)$ ist. Fallen einige oder alle $Q_{t}$ mit Punkten aus BK zusammen, so kann der Reduktionssatz auf die in Betracht kommenden ${ }^{\prime}{ }_{j}$ angewandt und es kann erreicht werden, dass $(k t+1)$ unter den Schnittpunkton ron BK verschieden sind ron den $Q_{i}$. Dann liegen aber im Innern mi ildestens eines der $\mathbf{T}_{j}$ mindestens $(k+1)$ Schnittpunkte aus BK. Gemäss Nr. 3.2.1. liegt also im Innern mindestens eines $T_{\text {, }}$ ein $k-S c h e i t e l$ im Widerspruch zur Annahme. Oder mindestens zn einem $\mathbf{T}_{1}$, etwa $T_{q}$, existiert eine $O C h \mathbf{K}^{\prime}$ so. dass ' $\mathbf{T}_{q} \mathbf{K}^{\prime}$ unendlich viele Punkte enthilt. Zufolge der Definition der $\mathbf{T}_{j}$ kann innerhalb ' $\mathbf{T}_{q}$ kein Häufungspunkt von $\mathbf{B} \mathbf{K}^{\prime}$ liegen. Es gibt aber cinen abg: schlossenen, kompakten Teilbogen $\mathbf{T}_{q}^{\prime}$ von $\mathbf{T}_{q}$, der mindestens $(k+1)$ Pankte ron $\mathbf{B} \boldsymbol{K}^{\prime}$ enthith und ganz im Innern von $\mathbf{T}_{q}$ liegt. Überdies muss ' $\mathbf{T}_{q}$ ' von endliehem $\mathrm{k}-\mathrm{OW}$ sein. Gemäss $\mathrm{Nr}$. 3.2.1 existiert aber dann ein $\mathbf{k}$-Scheitel in Innern von $\mathbf{T}_{q}$. Widerspruch.

3.2.1.2. Anmokung. In Nr. 2.2., Satz (B) (1), werden für die Anzahl der k-Scheitel des Grundbogens untere Schranken angegeben, die grösser sind als die in $\mathrm{Nr}$. 3.2.1.1. bestimmten; der Unterschied in den Voraussetzungen zwischen Nr. 2.2. (B) (1) und Nr. 3.2.1. besteht dabei darin, dass im letzteren Falle die eindeutige Existenz der k-Paratingente nicht (wie durch Vor. IV., Nr. 2.1.) gefordert wird. Es wird nun durch ein Beispiel gezeigt (fur $k=3$ und $t=1$ ), dass die Schranken in N. 3.2.1.1. im allgemeinen nicht verbessert werden können. Daraus folgt dann (Vgl. dazu Nr. 2.2., Zusatz):

Für die uneingeschränkte Gültigkeit des Satzes (1) in Nr. 2.2. (und insbesondere des Vierscheitelsa zes) ist die Vor. IVa. (Nr. 2.1.), d. h. die eindeutige Existenz der k-Scheitelparatingente, nicht zu entbehren (im speziellen Fall der zyklischen Ordung also nicht die Existenz des freien Scheitelkrummungskreises).

Beispiel eines Ovals vom zyklischen Ordnungswert Vier und mit nur zivei Scheiteln.

Konstruktion. Es sei $\mathbf{T}$ ein abgeschlossener Teilbogen eines Ellipsenquadranten, der keinen Sch itel enthält. Es sei $Q$ der Schnittpunkt der 
Halbtangenten an $\mathbf{T}$ in dessen Anfangs-und Endpunkt. Ferner sei $\mathbf{h}$ ein Kreis mit $Q$ als Zentrum, in dessen Inneren $\mathbf{T}$ liegt. Das Spiegelbild von $\mathbf{T}$ beztuglieh $\mathbf{h}$ ist dann wieder ein Bogen $\mathbf{S}$ rom zyklischen Ordnungswert Drei. Überdies enthält aber $\mathbf{S}$ einen Teilbogen $\mathbf{S}^{\prime}$ mit dem Endpunkt $E$ und dem Anfangspunkt $A$ derart, dass die Halbtangenten an $\mathbf{S}^{\prime}$ in $E$ und $A$ parallel sind. Spiegelt man nun $\mathbf{S}^{\prime}$ am Mittelpunkt $M$ der Strecke $A E$, so ist die Summe von $\mathbf{S}^{\prime}$ und seinem Spiegelbild $\mathbf{S}^{\prime \prime}$ ein Oral $\mathbf{O}$ mit überall stetiger Tangente. Es hat $\mathbf{C}$ genau $A$ und $E$ zu Scheiteln.

Beweis. Wie weiter unten gezeigt wird, ist der zyklische Ordnungswert von $\mathbf{C}$ gleich Vier, (wegen $k=3$ ) mithin $t=1$; gemäss Nr. 3.2.1.1. sind also mindestens. zwei Scheitel vorhanden; diese Scheitel können aber nur in $A$ und $E$ liegen, weil $\mathbf{S}^{\prime}$ and $\mathbf{S}^{\prime \prime}$ je den zyklischen Ordnungswert Drei besitzen. Dass nun der zyklische Ordnungswert von $\mathbf{C}$ gleich Vier ist, beweisen wir indirekt: Angenommen, es existiere ein Kreis $\mathbf{K}$, der mit $\mathbf{C}$ die 6 Punkte $P_{i}, i=1, \ldots, 6$, gemeinsam hat (der ayklische Ordnungswert von $\mathbf{C}$ ist höchstens gleich 6); die $P_{i}$ dürfen dabei o.B.d.A. sämtlich als Schnittpunkte angenommen werden $\left({ }^{35}\right)$. Da höchstens je drei der $P_{i}$ auf $\mathbf{S}^{\prime}$ bzw. auf $\mathbf{S}^{\prime \prime}$ liegen können, gibt es eine auf $\mathrm{C}$ natürliche Anordnung $P_{1}, \ldots, P_{6}$ der $P_{i}$ derart, dass, $P_{i} \varepsilon \mathbf{S}^{\prime}$ bzw. $P_{i} \in \mathbf{S}^{\prime \prime}$ je nachdem $i=1,2,3$, bzw. $i=4,5$, 6. Es sei dann $\mathrm{K}$ ebenfalls so orientiert, dass $P_{1}, \ldots, P_{6}$ eine natïrliche Anordnung auf $\mathrm{K}$ ist. Liegt nun eine untere Umgebung $\mathbf{V}_{1}$ von $P_{1}$ auf $K$ innerhalb (oder ausserhalb) $C$, so liegt eine untere Umgebung $\nabla_{4}$ von $P_{4}$ auf $\mathbf{K}$ ansserhalb (oder innerhalb) $\mathbf{C}$. Andererseits müssen aber $\mathbf{V}_{1}$ und $\mathbf{V}_{4}$ beide innerhalb oder beide ausserhalb $C$ liegen. In der Tat: Geht man vermöge Spiegelung an $M$ von $\mathbf{S}^{\prime \prime}$ zu $\mathbf{S}^{\prime}$ uber, so geht das Tripel $P_{4}, P_{5}, P_{6}$ in ein auf $\mathbf{S}^{\prime}$ natürlich geordnetes Tripel $P_{1}^{\prime} P_{2}^{\prime} P_{3}^{\prime}$ über, wobei $P_{i}^{\prime}$ Bild von $P_{3+i}$ ist und $P_{1}$ unterster unter den $P_{i}$ auf $\mathrm{S}^{\prime}$. Ferner wird ein, die $P_{4}, P_{5}, P_{6}$ sowie $\mathbf{V}_{4}$ enthaltender Kreisbogen gespiegelt in einen Kreisbogen $\mathbf{K}^{\prime}$ durch $P_{1}^{\prime}, P_{2}^{\prime}, P_{3}^{\prime}$ und es wird $\mathbf{V}_{4}$ gespiegelt in eine untere Umgebung $V_{1}^{\prime}$ von $P_{1}^{\prime}$ auf $\mathbf{K}^{\prime}$. Nun müssen alle Tripel auf $\mathbf{S}^{\prime}$ gleichartig liegen bezüglich $\mathbf{S}^{\prime}$, also bezüglich $\mathbf{C}$; denn dies gilt bekanntlich für jeden Teilbogen eines Ellipsenquadranten und $\mathbf{S}$ ist Spiegelbild am Kreis $h$ eines solchen T'eilbogens. Speziell müssen also $V_{1}$ und $V_{1}^{\prime}$ auf der gleichen Seite von $C$ liegen. Gleiches gilt dann aber für $V_{1}$ und $v_{\text {. }}$. Dies steht im Widerspruch mit den oben gemachten Feststellungen.

3.2.2. Jeder Häufungspunkt $\mathrm{H}$ von $\mathbf{k}$-Scheiteln des Grundbogens ist ein Punkt von mindestens dem $\mathrm{k}-\mathrm{OW}(\mathrm{k}+2)$. ( $\mathrm{Oh} \mathrm{n}$ e Vor. IVa., Nr. 2.1.).

Bew. Jedenfalls existiert eine Folge von $k-S c h e i t e l n$, die von einer Seite, z.B. von unten her gegen $H$ konvergiert. Da jeder k-Scheitel mindestens den $\mathbf{k - O W}(k+1)$ besitzt, ist somit jede untere Umgebung von $H$ auf B mindestens rom k-OW $(k+1)$. Weil es aber keine Punkté vom 
einseitigen k-OW $(k+1)$ gibt $\left({ }^{34}\right)$, muss $H$ mindestens den $\mathbf{k - O W}(k+2)$ besitzen, w.z.z.w.

3.2.3. Ein (echter oder unechter) Teilbogen $\mathbf{T}$ des Grundbogens $\mathbf{B}$ besitat dann und nur dann den $\mathrm{k}-\mathrm{OW} \mathrm{k}$, wenn in Innern von $\mathbf{T}$ kein $\mathbf{k}-S c h e i t e l$ liegt. (Ohne Vor. IVa., Nr. 2.1.).

Bew. Existiert mindestens ein k-Scheitel im Innern von 'T, so gibt es (gemäss der Definition des k-Scheitels) ein $(k+1)$-tupel $\gamma^{\prime}$, dessen Punkte sämtlich im Innern von $\mathbf{T}$ liegen; somit besitzt $\mathbf{T}$ mindestens den $\mathbf{k}-\mathrm{OW}$ $(k+1)$. Es enthalte andererseits $\mathbf{T}$ keinen $\mathbf{k}-$ Scheitel im Innern. Es genugt dann, za zeigen, dass jeder abgeschlossene, ganz im Innern von $\mathbf{T}$ gelegene und folglich kompakte Teilbogen $\mathbf{T}$ von $\mathbf{T}$ den k-OW $k$ besitzt; daraus folgt nämlich das Gleiche für 'T selbst (gemäss Nr. 3.1.1., Reduktionssatz). Jedenfalls ist ' $\mathbf{~ '}$ ' von endlichem $\mathbf{k}-\mathrm{OW}$, weil anderfalls eine $\mathrm{OCh} \mathbf{K}$ ' existierte derart, dass 'T K' unendlich viele Pankte und folglich (gemäss Nr. 3.2.1.) 'T im Innern einen k-Scheitel enthielte. Ist aber $\mathbf{T}$ von endlichem $k$-OW and ware der $k$-OW von $\mathbf{T}^{\prime}$ mindestens gleich $(k+1)$, so wurde wiederum aus Nr. 3.2.1. die Existenz mindestens oines Scheitels innerhalb $\mathbf{T}$ und damit ein Widerspruch sich ergeben.

3.2.4. Ein abgeschlossener Grundbogen B enthält in seinem Innern endlich viele k-Scheitel dann und nur dann, wenn $\mathbf{B}$ darstellbar ist als Summe endlich vieler abgeschlossener, bis auf ihre Begrenzungspunkte paarveise fremder Teilbogen von $\mathbf{B}$, deren jeder den $\mathrm{k}-\mathrm{OW} \mathrm{k}$ besitzt und deren im Innern von B gelegene Begrenzungspunkte genau die innerhalb $\mathbf{B}$ gelegenen $\left({ }^{32}\right) \mathbf{k}-$ Scheitel von B sind $\left.{ }^{33}\right)$. (Oh ne Vor. IVa., Nr. 2.1.).

Zusatz. Enthalt der Grandbogen B nur endlich viele k-Soheitel, so besitzt $\mathbf{B}$ einen beschränkten k-Ordnungswert.

Bew. Folgt aus N. 3.2.3.

\subsection{Beweis des Satzes (B) (4) in Nr. 2.2.}

Der Beweis dieses Satzes ergibt sich aus den folgenden Betrachtungen, die übrigens auch bei späteren Beweisen benötigt werden. Dabei wird stets auch die Vor. IVa. (Nr. 2.1.) als erfullt angenommen.

3.3.1. (a) Vor. Der Teilbogen $\mathbf{T}$ des Grundbogens $\mathbf{B}$ enthalte nur endlich viele $\mathbf{k}$-Scheitel; ferner enthalte $\mathbf{I}$ zwei $(\mathrm{k}+1)$-tupel, die gleichartig bzw.

(3i) Vgl, a. a. O. $\left({ }^{5}\right)$, Nr. 6.3.

(32) Die Begrenzungspunkte (Anfangs:und Endpunkt) fron B könnon nicht k-Scheitel sein, weil sie Begrenzungspunkte von Bogen des $k-O W k$ sind.

( $\left.{ }^{33}\right)$ Grundpunkte nehmen dabei keine Sonderstellung ein und dürfen mit k-Scheiteln zusammenfallen. 
ungleichartig sind und deren Anfangspunkte auf $\mathbf{T}$ durch eine ungerade bzwo. gerade Anzahl Grundpunkte (die als Schnittpunkte mit den $(\mathrm{k}+1)$-tupeln ver. bunden sind) von einander getrennt werden $(\mathrm{k} \geq 1)$.

Beh. In Innern von $\mathbf{T}$ liegen mindestens zwei $\mathbf{k}-$ Scheitel.

(b) Vor. Der Teilbogen $\mathbf{T}$ von $\mathbf{B}$ enthalte nur endlich viele $\mathbf{k}-$ Scheitel.

Beh. (I) Ist der (notvendig beschräntite) k-OW von $\mathbf{T}$ nicht kleiner als $(\mathrm{k}+2)$, so enthält $\mathbf{T}$ in Innern mindestens zwei $\mathbf{k}-$ Scheitel.

(II) Enthält $\mathbf{T}$ in seinem Inneren genau einen $\mathbf{k}-$ Scheitel, so besitzt $\mathbf{T}$ den $\mathbf{k - O W}(\mathbf{k}+1)$.

Bew. (a) Gemăss Nr. 3.2.4., Zusatz, besitzt $\mathbf{T}$ beschränkten k-OW. Es seien dann $\gamma=\left(S_{1}, \ldots, S_{k+1}\right)$ und $\gamma^{\prime}=\left(S_{1}^{\prime}, \ldots, S_{k+1}^{\prime}\right)$ die beiden $(k+1)$-tupel, wobei etwa $S_{1}$ unterhalb $S_{1}^{\prime}$ liege. Zufolge Nr. 3.2.1. entspricht dem $\gamma$ bzw. $\gamma^{\prime}$ je (mindestens) ein k-Scheitel $A$ bzw. $A^{\prime}$. Wir zeigen, dass $A=A^{\prime}$ zu einem Widerspruch mit Vor. IVa., Nr. 2.1., führt, d. h. zur Existenz zweier ungleichartiger k-Scheitelparatingenten in $A=A^{\prime}$. Es sei also $A=A^{\prime}$. Da bei den auf $\gamma$ bzw. $\gamma^{\prime}$ auszuübenden Kontraktionsprozessen sich $S_{1}$ und $S_{1}^{\prime}$ beide nach oben hin bewegen, liegt $A$ oberhalb $S_{1}^{\prime}$. Es sei ferner $S_{1}$ von $S_{1}^{\prime}$ auf $\mathbf{B}$ durch $t$ Grundpunkte $G_{i}{ }^{\prime}, \ldots, G_{t}{ }^{\prime}$ (diese natürlich angeordnet) getrennt und weiter $G_{t}{ }^{\prime}$ von $A$ durch $s$ Grundpunkte $G_{1}^{\prime \prime}, \ldots, G_{s}{ }^{\prime \prime}$ (ebenfalls in natürlicher Anordnung), $t \geq 0, s \geq 0$. Im Laufe des Kontraktionsprozesses (vgl. die Verschärfung des Kontraktionssatzes in $\mathrm{Nr}$. 3.1.3.) überschreitet $S_{1}$ zunächst die $G_{1}{ }^{\prime}, \ldots, G_{t}{ }^{\prime}$; dabei geht $\gamma$ uber in ein mit $\gamma$ gleichartiges oder ungleichartiges $(k+1)$-tupel $\gamma^{\prime \prime}=\left(S_{1}^{\prime \prime}, \ldots, S_{k+1}^{\prime \prime}\right)$ je nachdem $t \equiv 0$ oder $t \equiv 1(\bmod 2)$ ist. Andererseits ist nach Vor. $\gamma$ gleich-bzw. ungleichartig mit $\gamma^{\prime}$ je nachdem $t \equiv 1$ oder $t \equiv 0$ (mod 2). Somit sind $\gamma^{\prime \prime}$ und $\gamma^{\prime}$ stets ungleichartig. Und diese Ungleichartigkeit von $\gamma^{\prime \prime}$ and $\gamma^{\prime}$ bleibt im weiteren Verlaufe des Kontraktionsprozesses fur $\gamma^{\prime \prime}$ bzw. beim Kontraktionsprozess für $\gamma^{\prime}$ erhalten, weil $\mathrm{ja} S_{\mathrm{t}}{ }^{\prime \prime}$ und $S_{\mathrm{t}}{ }^{\prime}$ beide die nämlichen Grundpunkte nach oben hin überschreiten (und sonst keine Grund. punkte), bevor sie sich dem Punkte $A$ (beliebig) nähern. Somit sind die aus der Kontraktion von $\gamma$ und von $\gamma^{\prime}$ resultierenden k-Scheitelparatingenten $\mathbf{P}$ bzw. $\mathbf{P}^{\prime}$ an $\mathbf{B}$ in $A$ ungleichartig. Dies und somit die Annahme, dass $A=A^{\prime}$, steht aber im Widerspruch zum Eindeutigkeitsaxiom IVa. in Nr. 2.1.

(b). Die Beh. (b) (I) folgt aus der Tatsache, dass die aus einem ununterbrochenen $(k+2)$-tupel $\left(S_{1}, \ldots, S_{k+2}\right)$ sich ergebenden $(k+1)$-tupel $\left(S_{1}, \ldots, S_{k+1}\right)$ und $\left(S_{2}, \ldots, S_{k+2}\right)$ gleich-bzw. ungleichartig sind je nachdem $S_{1}$ und $S_{2}$ auf $\mathbf{B}$ durch eine ungerade bzw. gerade Anzahl Grundpunkte getrennt werden, die sämtlich als Schnittpunkte von $\mathbf{B}$ mit der in Betracht kommenden OCh angenommen werden dürfen. Aușil(I) folgt dann (II).

3.3.2. Aus Nr. 3.3.1. Beh. (b) (II) ergibt sich bei Vor. IVa. noch die folgende

Verschärung des Satzes in Nr. 3.2.4.: Jeder isolierte k-Scheitel 
(im Innern) des Grundbogens $\mathbf{B}$ ist ein minimaler $\mathbf{k}-$ Scheitel, d.h. jeder iso. lierte $\mathbf{k}-$ Scheitel besitzt den (minimalen) $\mathrm{k}-\mathrm{OW}(\mathrm{k}+1)$.

3.3.3. Aus Nr. 3.3.1., (a), folgt weiter:

Enthält der Grundbogen $\mathbf{B}$ in seinen Innern genau einen $\mathbf{k}-$ Scheitel $\mathrm{S}$, so liegen je zwei, in Innern von $\mathrm{B}$ enthaltene $(\mathrm{k}+1)$-tupel gleichartig oder ungleichartig, je nachdem ihre Anfangspunkte durch eine gerade bzw. ungerade Anzahl von Grundpunklen auf $\mathbf{B}$ von einander getrennt sind.

Anmerkung. Dabei liegen die Anfangspunkte bzw. die Endpunkte aller $(\mathrm{k}+1)$-tupel unterhalb bzw. oberhalb des $\mathbf{k}-$ Scheitels $\mathrm{S}$; diese Anfangspunkte z.B. werden also durch $S$ nicht von einander getrennt. Es ist daher auf die Gultigkeit des Satzes ohne Einfluss, ob $S$ Grundpunkt ist oder nicht.

Bew. Andernfalls existierten nämlich zwei $(k+1)$-tupel, die der Voraussetzung des Satzes in Nr. 3.3.1. (a) genugten.

3.4. Beweis von Satz (B) (1) in Nr. 2.2.

Es sollen die Vor. I. -I V. (Nr. 2.1.) gelten.

3.4 1. Vor. Der Grundbogen B besitze nur endlich viele $\mathbf{k}-$ Scheitel. Ferner sei A ein innerer Punkt von B, aber kein k-Grundpunkt. Das System aller OCh aus $\mathrm{k}$, welche durch Punkt $\mathrm{A}$ gehen, sei $\mathbf{k} *$.' Weiter seien $\mathrm{Q}^{\prime}$ und $\mathrm{Q}^{\prime \prime}$ zwei, von $\mathrm{A}$ verschiedene $\mathrm{k}^{*}$-Scheitel innerhalb $\mathbf{B}$, wobei $\mathrm{Q}^{\prime}$ zwischen $\mathrm{A}$ und $\mathrm{Q}^{\prime \prime}$ liege. Die Existenz weiterer $\mathrm{k}^{*}-$ Scheitel zwischen $\mathrm{A}$ und $\mathrm{Q}^{\prime}$, sowie zwischen $\mathrm{Q}^{\prime}, \mathrm{Q}^{\prime \prime}$ ist nicht ausgeschlossen; ferner können $\mathrm{Q}^{\prime}, \mathrm{Q}^{\prime \prime}$ sonie $\mathrm{A}$ auch $\mathbf{k - S c h e i t e l ~ s e i n ~ u n d ~} \mathrm{Q}^{\prime}, \mathrm{Q}^{\prime \prime}$ auch $\mathbf{k}-$ Grundpunkte.

Beh. (I) Es existiert

(1) zwischen $\mathrm{A}$ und $\mathrm{Q}^{\prime}$, den Punkt $\mathrm{Q}^{\prime}$ eingeschossen, mindestens ein $\mathrm{k}-$ Scheitel $\mathrm{S}^{\prime}$, velcher übrigens stets von A verschieden ist; dagegen kann $\mathrm{S}^{\prime}=\mathrm{Q}^{\prime}$ sein.

(2) zwischen $Q^{\prime}$ und $Q^{\prime \prime}$, den Punkt $Q^{\prime \prime}$ eingeschlossen aber nicht $Q^{\prime}$, mindestens ein $\mathrm{k}-\mathrm{S}^{\mathrm{s} c h}$ eitel $\mathrm{S}^{\prime \prime}$, der übrigens von $\mathrm{Q}^{\prime \prime}$ verschieden ist, falls nicht $\mathrm{Q}^{\prime}$ und $\mathrm{Q}^{\prime \prime}$ beide $\mathrm{k}-$ Scheitel sind.

(II) Ist die Anzahl der $\mathbf{k}-S c h e i t e l$ von $\mathbf{B}$ gleich q, so ist die Anzahl der $\mathrm{k} *$-Scheitel von $\mathbf{B}$ (ebenfalls endlich und zmar) nicht grösser als q.

Bew. Es sei $\mathbf{T}$ der, von $A$ und $Q^{\prime \prime}$ begrenzte, $Q^{\prime}$ enthaltende Teilbogen von B, wobei $\mathbf{T}$ o.B.d.A. den Anfangspunkt $A$ besitze. Ferner sei $\mathbf{S}$ derjenige Teilbogen von B, der $Q^{\prime}$ als Anfangs=und $Q^{\prime \prime}$ als Endpunkt besilzt. Schliesslich sei $\mathbf{U}^{\prime}$ bzw. $\mathbf{U}^{\prime \prime}$ eine Umgebung von $Q^{\prime}$ bzw. von $Q^{\prime \prime}$ auf $\mathbf{B}$, in der weder $A$ noch $Q^{\prime \prime}$ bzw. weder $A$ noch $Q^{\prime}$ enthalten ist und in der kein k-Scheitel liegt ausgenommen höchstens $Q^{\prime}$ bzw. $Q^{\prime \prime}$. Zufolge der Vor. existieren derartige, sogar zu einander fremde, $\mathbf{U}^{\prime}$ und $\mathbf{U}^{\prime \prime}$.

Betr. Beh. (I) (1). Da $Q^{\prime}$ ein $\mathbf{k}^{*}$-Scheitel ist, existiert ein in $\mathbf{U}^{\prime}$ enthaltenes $k$-tupel $\gamma=\left(S_{2}, \ldots, S_{k+1}\right)$, mit dem $A$ verbunden ist. Es sei $A^{\prime}$ der am nächsten unterhalb $S_{2}$ gelegene, mit $\gamma$ verbundene Punkt, wobei $A=A^{\prime}$ zuge. 
lassen ist. Erforderlichenfalls kann nun (gemäss Nr. 3.1.1., Reduktionssatz) durch eine beliebig kleine Änderung der zu $\gamma$ gehörigen OCh stets erreicht werden, dass $A^{\prime}$ im Innern von $\mathbf{T}^{\prime}=(\mathbf{T}-\mathbf{S})+\mathbf{U}^{\prime}$ liegt und Schnitpunkt ist, wahrend zugleich $S_{2}, \ldots, S_{k+1}$ Schnittpunkte sind. Damit ist die Existenz eines $(k+1)$-tupels innerhalb ' $\mathbf{T}$ ' nachgewiesen und folglich die eines $\mathbf{k}-$ Scheitels $S^{\prime}$ innerhalb $\mathbf{T}^{\prime}$. Ist $Q^{\prime}$ selbst k-Seheitel, so kann $S^{\prime}=Q^{\prime}$ sein, andernfalls liegt $S^{\prime}$ innerhalb $\left(\mathbf{T}^{\prime}-\mathbf{U}^{\prime}\right)$ gemäss der Definition ron $\mathbf{U}^{\prime}$, w.z.z.w.

Betr. Beh. (I) (2). Nach Vor.' existiert je ein in $\mathbf{U}^{\prime}$ bzw. in $\mathbf{U}^{\prime \prime}$ enthaltenes (naturlich geordnetes) $k$-tuipel $\gamma^{\prime}=\left(S_{1}^{\prime}, \ldots, S_{k}{ }^{\prime}\right)$ bzw. $\gamma^{\prime \prime}=\left(S_{1}^{\prime \prime}, \ldots, S_{k}{ }^{\prime \prime}\right)$, mit dem $A$ verbunden ist. Alle Punkte von $\gamma^{\prime}$ liegen unterhalb aller Punkte von $\gamma^{\prime \prime}$. Wir unterscheiden nun folgende drei, alle Möglichkeiten erschöpfende Falle: (a) es ist $\mathrm{Q}^{\prime}$ kein $\mathbf{k}-$ Scheitel; (b) es ist $\mathrm{Q}^{\prime \prime}$ kein $\mathrm{k}-$ Scheilel; (c) es sind $\mathrm{Q}^{\prime}$ und $\mathrm{Q}^{\prime \prime}$ beide $\mathbf{k}-$ Scheitel.

Betr. Fall (a). Nach Annahme enthält $\mathbf{U}^{\prime}$ keinen k-Scheitel. Zwecks indirekten Beweises der Beh. setzen wir uberdies voraus, dass auch im Innern von $\mathbf{S}$ keine k-Scheitel liegen. Wir unterscheiden znei Unterfälle (a') und $\left(a^{\prime \prime}\right)$; nämlich :

Unterfall $\left(\mathbf{a}^{\prime}\right)$ : Es sei auch $\mathrm{Q}^{\prime \prime}$ kein $\mathbf{k}-$ Scheitel. Dann besitzt $\mathbf{R}=\mathbf{U}^{\prime}+\mathbf{S}+\mathbf{U}^{\prime \prime}$ den k-OW $k$ gemäss $\mathrm{Nr}$. 3.2.3. Wir halten $S_{2}{ }^{\prime \prime}, \ldots, S_{k}{ }^{\prime \prime}$ fest und ändern das natürlich geordnete $(k+1)$-tupel $\left(A, S_{1}{ }^{\prime \prime}, \ldots, S_{k}{ }^{\prime \prime}\right)$ so ab, dass $S_{1}^{\prime \prime}$ monoton nach unten (gegen $A$ ) hin sich bewegt; an Stelle von $A$ erhalten wir dann Punkte oberhalb (des ursprünglichen Ortes von) $A$. Da $\mathbf{R}$ den k-OW k besitzt, können in $\mathbf{R}$ bei unserer Änderung keinerlei Gewinne stattfinden, solange $S_{1}^{\prime \prime}$ oder vielmehr der aus. $S_{1}^{\prime \prime}$ durch die Änderung hervorgehende Punkt sich innerhalb $\mathbf{R}$ befindet. Mán kann daher durch die monotone Änderung $S_{1}^{\prime \prime}$ in $S_{1}{ }^{\prime}$ uberführen und dadurch aus dem $k$-tupel $\gamma^{\prime \prime}$ das $k$-tupel $\left(S_{1}^{\prime}, S_{2}^{\prime \prime}, \ldots, S_{k}{ }^{\prime \prime}\right)$ erhalten. Mit dem letzteren ist aber der urspı tingliche Ort von $\dot{A}$ nicht mehr verbunden, da er bei der Änderung ins Innere eines wachsenden Teilbogens $W$ gerät; verbunden ist mit $\left(S_{1}^{\prime}, S_{2}{ }^{\prime \prime}, \ldots, S_{k}{ }^{\prime \prime}\right)$ hingegen ein Punkt, der oberhalb des obersten, unterhalb $\mathbf{R}$ gelegenen und mit $\gamma^{\prime \prime}$ verbundenen Punktes $A^{\prime \prime}$ liegt, aber nicht zu $\mathbf{R}$ gehört. Die soeben für $S_{1}^{\prime}, S_{1}^{\prime \prime}$ angestellten Überlegungen können für $S_{z}{ }^{\prime}, S_{2}{ }^{\prime \prime}$ (bei festen $S_{1}{ }^{\prime}, S_{3}{ }^{\prime \prime}, \ldots, S_{k}{ }^{\prime \prime}$ ) wiederholt werden. Da hierbei die monotone Bewegung der mit dem jeweils betrachteten $k$-tapel verbundenen Punkte stets im gleichen Sinne verläuft wio beim ersten Schritt (für $S_{1}{ }^{\prime}, S_{1}{ }^{\prime \prime}$ ), so wird $\mathbf{W}$ bei diesem zweiten Schritt weiter wachsen, also der ursprüngliche Ort von $A$ insbesondere mit $\left(S_{1}^{\prime}, S_{2}^{\prime}, S_{3}{ }^{\prime \prime}, \ldots, S_{k}{ }^{\prime \prime}\right)$ nicht verbunden sein. Durch Fortsetzung dieses Verfahrens für $S_{3}^{\prime}, S_{3}^{\prime \prime}$ usw. gelangen wir zum Ergebnis: Es ist $\gamma^{\prime \prime}$ in $\gamma^{\prime}$ derart uberfuhrbar, dass der ursprüngliche Ort von $A$ mit keinem der bei der Überfuhrung auftretenden, innerhalb $\mathbf{R}$ gelegenen $k$-tupel verbunden ist, insbesondere also nicht mit $\gamma^{\prime}$. Widerspruch mit der Ànnahme über $\gamma^{\prime}$.

Unterfall (a"). Es sei $\mathrm{Q}^{\prime \prime}$ aber nicht $\mathrm{Q}^{\prime}$ ein k-Kcheitel (und das Innere 
von $\mathbf{S}$ sei frei von $\mathbf{k}-$ Scheitelnj. Wir versuchen, $\gamma^{\prime \prime}$ durch den gleichen monotonem Prozess wie im Unterfall $\left(a^{\prime}\right)$ in $\gamma^{\prime}$ überzuführen. Dabei ergibt sich aber die Schwierigkeit, dass Gewinne von Punkten innerhalb $\mathbf{R}$ unterhalb des jeweils (nach unten) bewegten Punktes $S_{*}^{\prime \prime}$ nicht von vorneherein ausgeschlossen sind $(x=1, \ldots, k)$, weil $\mathbf{R}$ jetzt den $\mathbf{k}-\mathrm{OW}(k+1)$ besitzt (gemass Nr. 3.3.1., (b) (II)). Unter Bonutzung der vorausgesetzten Eindeutigkeit der k-Paratingenten (Nr. 2.1., IV.) kann man diese Schwierigkeit folgendermassen umgehen. Wir führen zunächst $\gamma^{\prime \prime}$ durch den im Unterfall $\left(a^{\prime}\right)$ verwendeten monotonen Prozess in ein unterhalb $Q^{\prime \prime}$ gelegenes $k$-tupel $\bar{\gamma}^{\prime \prime}$ über und stützen uns auf die (weiter unten zu beweisende) $\mathrm{ZW}$ is chen behauptung: "Werden (was stets möglich ist) $\bar{\gamma}^{\prime \prime}$ und $\gamma^{\prime \prime}$ beide nar hinreichend nahe bei $Q^{\prime \prime}$ gewählt, so finden im Verlauf unserer Überführung von $\gamma^{\prime \prime}$ in $\bar{\gamma}^{\prime \prime}$ keine Gewinne von Punkten innerhalb $\mathbf{R}^{\prime}=\mathbf{U}^{\prime}+S$ statt. $\gg$ Weil aber $\mathbf{R}^{\prime}$ den $\mathbf{k}-\mathrm{OW} \mathbf{k}$ besitzt, lässt sich auf die beiden, innerhalb $\mathbf{R}^{\prime}$ gelegenen $k$-tupel $\overline{\gamma^{\prime \prime}}$ und $\gamma^{\prime}$ der gleiche (monotone) Überführungsprozess mit dem gleichen Ergebnis anwenden wie im Unterfall $\left(a^{\prime}\right)$; dabei ist nnr noch zu beachten, dass (der ursprüngliche Ort des Punktes) $A$ bei der Überfuhrung von $\gamma^{\prime \prime}$ in $\bar{\gamma}^{\prime \prime}$ ins Innere eines vachsenden Bogens $\mathbf{W}$ ger:t und dass $\mathbf{W}$ auch bei der (monotonen) Überführung von $\bar{\gamma}^{\prime \prime}$ in $\gamma^{\prime}$ ohne Unterbrechung weiter nächst. Die Annahme, das Innere von $\mathbf{S}$ sei frei von $\mathrm{k}$-Scheiteln, fiihrt somit auch im Unterfall $\left(a^{\prime \prime}\right)$ zu einem Widerspruch. Wir haben jetzt noch den Beweis der Zwischenbehauptung nachzuholen; dabei ist nach wie vor vorausgesetzt, dass $\mathbf{S}$ frei ist von $\mathbf{k - S c h e i -}$ teln. Wir bemerken vorerst, dass mit $\gamma^{\prime}$ noch ein oberhalb $S_{k}^{\prime \prime}$ sowie innerhalb $\mathbf{U}^{\prime \prime}$ gelegener Punkt $S^{\prime \prime}{ }_{k+1}$ verbunden sein kann; dieser bewegt sich aber bei der (monotonen) Überführnng von $\gamma^{\prime \prime}$ in $\gamma^{\prime \prime}$ stets nach oben (solange er nicht etwa oberhalb $\mathbf{U}^{\prime \prime}$ verloren geht), kann also beim Beweise der Zwischenbehauptung ausser Betracht bleiben. Wir führen diesen Beweis indirekt, nehmen also an, es würden bei unserer Überfuhrung von $\gamma^{\prime \prime}$ in $\bar{\gamma}^{\prime \prime}$ Gewinne innerhalb $\mathbf{R}^{\prime}$ stattfinden. Da $\mathbf{R}=\mathbf{U}^{\prime}+\mathbf{S}+\mathbf{U}^{\prime \prime}$ den $\mathbf{k}-\mathrm{OW}(k+1)$ besitzt, können Gewinne unterhalb des jeweils (nach unten) bewegten Punktes $S_{x}{ }^{\prime \prime}$ nur dann stattfinden, wenn $S^{\prime \prime}{ }_{k+1}$ nicht vorhanden bzw. schon nach oben hin aus $\mathbf{U}^{\prime \prime}$ herausgerückt ist. . Es kann auch nur höchstens ein Punkt $P$ innerhalb: $\mathbf{R}^{\prime}$ gewonnen. werden und zwar nur derart, dass $P$ monoton nach oben hin sich bewegend über den Anfangspunkt $U^{\prime}$ von $\mathbf{U}^{\prime}$ hinweg ins Innere von $\mathbf{R}^{\prime}=\mathbf{U}^{\prime}+\mathbf{S}$ hinein und dem $S^{\prime \prime}{ }_{\text {}}$ monoton entgegenrückt. Da aber $\mathbf{R}^{\prime}$ den $\mathbf{k - O W} k$ besitzt (gemäss Nr. 3.2.3.), kann mit $\overline{\gamma^{\prime \prime}}$ kein Punkt in $\mathbf{R}^{\prime}$ verbunden sein. Daher müsste $P$ noch vor Vollendung der Überfürung von $\gamma^{\prime \prime}$ in $\bar{\gamma}^{\prime \prime}$ den ganzen Bogen $\mathbf{R}^{\prime}-\mathbf{U}^{\prime \prime}$ durchlaufen und nach $\mathbf{U}^{\prime \prime}$ hineingelangen, um verloren zu gehen. Wäre also die Zwischenbehauptung falsch, so existierte zu jedem Punkt $X$ einer Umgebung $\mathbf{V}^{\prime}$ von $U^{\prime}$ auf $\mathbf{R}^{\prime}-\mathbf{U}^{\prime \prime}$ in jeder (beliebig kleinen) Umgebung $\mathbf{Z}$ von $Q^{\prime \prime}$ auf $\mathbf{B}$ ein $k$-tupel $\tau$ derart, dass $X$ mit $\tau$ verbunden ist. Daher wäre $X$ in der (gemäss Vor. IV. (Nr. 2.1.) eindeutig bestimmten) $\mathbf{k}-$ Paratingente $\mathbf{P}$ 
an $B$ in $Q^{\prime \prime}$ enthalten. Somit liage der ganze Bogen $\mathbf{V}^{\prime}$ auf $\mathbf{P}$, während doch BP als nirgends dicht anf $B$ vorausgesetzt ist (Nr. 2.1., IV.). Damit ist die Zwischenbehauptung (indirekt), also die Beh. (I) (2) auch für den Unterfall $\left(a^{\prime \prime}\right)$ und damit für den Fall $(a)$ bewiesen.

Betr. Fall (b). Der Beweis kann wie im Falle (a) gefithrt werden, wenn man nur $g^{\prime}$ und $g^{\prime \prime}$ ihre Rollen tauschen lässt.

Betr. Fall (c). Fur die Beh. (I) (2) ist in diesem Falle nichts zu beweiken.

Betr. Beh. (II) Die Anzahl der k*-Suheitel, die unterhalb bzw. oberhalb $A$ liegen (wenn wir hier $\mathbf{B}$ als Bogen voraussetzen, was die zu beweisend. Aussage nicht beeinträ(chtigt), sei mindestens $s$ bzw. $t$ mit $0 \leq s, 0 \leq t$, $s+t=p \geq 1$. Gemäss Beh. ( $\mathrm{I}_{1}$ ist die Anzahl der k-Scheitel unterhalb bzw. oberhalb $A$ dann mindestens gleich $s$ bzw. $t$. Sind namlich etwn $R_{1}, \ldots, R_{t}$ oberhalb $A$ gelegene $\mathbf{k} *-$ Scheitel in nattirlicher Anordnung und setzt man nooh $R_{0}=A$, so liegt mindestens ein $\mathbf{k}$-Scheitel innerhalb des Bogens mit $R_{\tau-1}$ bzw. $R_{\tau}$ als Anfangs- bzw. als Endpunkt oder in dessen Endpunkt $R_{\text {; }}$, $\tau=1, . ., t$. Entsprechend schliesst man fïr die unterhalb $A$ gel genen $k-S c h e i-$ tel, woraus $s+t \leq q$ folgt, w.z.z.w.

A mmerkung. Die beim Beweise des vorstehenden Satzes verwondeto Methode zeigt gewisse Analogien znm Juelschen Kosrespondenzprinzip ${ }^{34}$ ).

3.4.2. Besitat $\mathrm{B}$ mindestens den $\mathrm{k}-\mathrm{OW} \mathrm{p}$ mit $\mathrm{k}+1 \leq \mathrm{p}$, so ist die Anzahl $\mathrm{q}$ der $\mathbf{k}-$ Scheitel auf $\mathbf{B}$ mindestens gleich $(\mathrm{p}-\mathrm{k})$. Ist $\mathbf{B}$ speziell eine Kurve $\mathbf{C}$, so ist sogar $\mathrm{q} \geq \mathrm{p}$.

Bew. 0.B.d.A können wir ausnahmslos annehmen, dass der zu betrachterde Grundbogen (Grundkurve) nur endlich viele k-Scheitel enthalt. Dann verwenden wir vollstandige Induktion. Die Beh. ist richtig aunächst für $\mathrm{k}=1$. In der Tat: Es sei $\mathbf{K}$ eine $\mathrm{OCh}$, die mit $\mathbf{B}$ (mindestens) $p$ Schnittpunkte $S_{1}, \ldots, S_{p}$ (in naturlicher Anordnung auf B) gemeinsam bat. Wir können o.B.d.A. (gemass Nr. 2.1.1., Reduktionssatz) $\left(S_{1}, \ldots, S_{p}\right)$ als ununterbrochenes p-tupel annehmen (gemäss Nr. 2.2. und 3.1.1. sind Grundpunkte unter die $S_{1}, \ldots, S_{p}$ nicht aufzunehmen). Wendet man auf das 2-tnpel $\left(S_{p}, S_{q+1}\right)$, $\rho=1, \ldots, p-1$, den Kontraktionssatz an, so ergibt sich die Existenz eines k-Scheitels, der im Innern des Bogens mit dem Anfargs- bzw. Endpunkt $S_{q}$ bzw. $S_{p+1}$ liegt (das Vorhandensein von Grundpunkten spielt beim Existenzbeweis für diese $\mathbf{k}$-Scheitel keine Rolle). Somit existieren mindestens $(p-1)$ k-Scheitel auf B, die sämtlich z.B. von $S_{1}$ ver schieden sind. Ist speziell $\mathbf{B}$ eine Kurve, so enthält auch der Bogen mit $S_{p}$ als Anfangs- und $S_{\text {: als }}$ Endpunkt in seinem Innern einen k-Scheitel; die Gesamtzahl der k-Scheitel in Falle einer Grundkurve ist daher mindestens gleich $p$.

${ }^{(34)} \mathrm{Vgl.} \mathrm{JUEL,} \mathrm{C.,} \mathrm{Einleitung} \mathrm{in} \mathrm{die} \mathrm{Theorie} \mathrm{den} \mathrm{ebenen} \mathrm{Elementarkureen} \mathrm{3.} \mathrm{und} \mathrm{4.} \mathrm{Orrlnung,}$ "Danske Vidensk. Selsk. Skrifter, 7. R., naturv. og mathem. Afd. ", X1. 2 (1914). 
Induktionsannahme: Die Beh. sei schon für jedes $k$ mit $1 \leq k \leq k^{\prime}$ bewiesen. Es sei $k^{\prime \prime}=k^{\prime}+1$, ferner sei $\mathbf{k}^{\prime \prime}$ ein System von OCh mit der Grundzahl $k^{\prime \prime}$ und es besitze $\mathbf{B}$ den $k^{\prime \prime}-0 \mathrm{~W} p$ mit $k^{\prime \prime}+1 \leq p$. Demgemäss sei $\gamma=\left(S_{1}, \ldots, S_{p}\right)$ ein $p$-tupel auf $B$. Durch eine beliebig kleine Abanderung der $S_{\text {, }}$ in $\gamma$ kann übrigens (da $\mathbf{B}$ beschränkten k-OW besitzt, Nr. 3.2.4., Zusatz) stets erreicht werden $\left({ }^{3 i}\right)$, "dass keines der $S_{\varphi}$ ein $\mathbf{k}^{\prime \prime}$-Scheitel ist und dass ïberdies $S$, auf keiner der (endlich vielen) $\mathbf{k}^{\prime \prime}$-Scheitelparatingenten von $\mathbf{B}$ liegt; die letztere Forderung ist deshalb erlüllbar, weil der Durchschnitt einer jeden der endlich vielen $\mathbf{k}^{\prime \prime}$-Scheitelparatingenten mit $\mathbf{B}$ nirgends dicht auf B sein sollle (Nr. 2.1, IV.). Nun bildet aber die Gesamtheit der den Punkt $S_{1}$ enthaltenden OCh $\mathbf{K}$ von $\mathbf{k}^{\prime \prime}$ ein System $\mathbf{k}^{\prime}$ von OCh mit der Grundzahl $k^{\prime}=k^{\prime \prime}-1$; (z:ıfolge der Wahl von $S_{1}$ sind iibrigens die $\mathbf{k}^{\prime \prime}-$ Scheitel sämtlich verschieden. von den $k^{\prime}$-Scheiteln) anch ist $S_{1}$ selbst kein $\mathbf{k}^{\prime}$-Scheitel (gemäss der Festsetzung zu Beginn der Nr. 2.2.). Nach Induktionsannahme ist nun die Anzahl der $\mathbf{k}^{\prime}$-Scheitel mindestens $(p-1)-k^{\prime}=p-k^{\prime \prime}$. Gemiss Nr. 3.4.1., Beh. (II), ist somit die Anzahl der $\mathbf{k}^{\prime \prime}-$ Scheitel nicht kleiner als $\left(p-k^{\prime \prime}\right)$; w.z.z.w. Ist speziell $\mathbf{B}$ eine Kurve $\mathbf{C}$, so ist nach der Induktionsannahme die Anzahl $\cdot m$ der $\mathbf{k}^{\prime}$-Scheitel $T_{\mu}^{\prime}, \mu=1, \ldots, m$, nicht kleiner als $(p-1)$; dabei zithlt $S_{t}$ nicht zu den $T_{\mu}^{\prime}$ (vgl. Nr. 2.2., Festsetzung). 0.B.d.A. sei $T_{1}^{\prime}, \ldots, T_{m}{ }^{\prime}$ eine natïrliche Anordnung der $T_{\mu}^{\prime}$ anf $\mathbf{C}$ derart, dass $S_{1}$ unterhalb $T_{i}^{\prime}$ liegt. Znfolge der Wahl von $S_{1}$ fällt keiner der $\mathbf{k}^{\prime \prime}$-Scheitel in einen der Punkte $T_{\mu}^{\prime}$. Gemäss Nr. 3.4.1., Beh. (I), liegen dann im Innern des; $T_{1}^{\prime}$ enthaltenden, $S_{1}$ als Anfangs- und $T_{m}^{\prime}$ als Endpunkt besitzenden T'eilb)gens von $\mathbf{C}$ nicht weniger als $m \mathbf{k}^{\prime \prime}$-Scheitel; ebenso liegt im Innern des, $T_{1}^{\prime}$ nicht enthaltenden Bogens mit $T_{m}^{\prime}$ als Anfangs- und mit $S_{1}$ als Endpunkt mindestens ein $\mathbf{k}^{\prime \prime}$-Scheitel. Zusammen sind also nicht weniger als $m+1 \geq(p-1)+1=p$ solche $\mathbf{k}^{\prime \prime}-$ Scheitel vorhanden; w.z.z.w.

\section{3.̌. Beweis von Satz (B) (2) in Nr. 2.2.}

Es gelten die Vor. I.-IVa. (Nr. 2.1.) Wir bemerken zunächst, dass die k-Scheitel isoliert auf $\mathbf{B}$ liegen; denn gemäss Nr. 3.2.2. ist der $\mathbf{k}-\mathrm{OW}$ eines jeden Hufungspunktes vọ $\mathbf{k - S c h e i t e l n ~ m i n d e s t e n s ~ g l e i c h ~}(k+2)$; jeder kompakte Teilbogen von $\mathbf{B}$ enthält somit nur endlich viele k-Scheitel. Während nun Satz (B) (1) in Nr. 2.2. die Anzahl der k-Scheitel nach unten hin beschrinkte, tut dies Satz (2) nach oben hin, allerdings nur $\left({ }^{36}\right)$ für die Grund-

3i) Vgl. a. a. (). (5), Nr. 1.8., S. 16.

$\left.{ }^{(30}\right)$ Es gibt z. B. Bogen vom zyklischen Ordnungswert $t \geq 5$ mit unendlich vielen l'unkten von mindestens dem zyklischen $O W$ Vier. (Vgl. Haupr, Raumbogen beliebig vorge. gebener lokaler Ordnung, "Journ. f. d. r. u. angew. Math.", 184j(1942), S. 77 ff., ferner - Sitz. Ber. d. bayer. Akad. d. Wiss. math. - naturw. Abt.", 1939, S. 253 ff). Daraus folgt, dass für Bogen (oder Kurven) von einem $\mathrm{k}-\mathrm{OW}$ grösser als $(k+1)$ in allgemeinen eine (endliche) Schranke fir die Anzahl der k-Seheitel nicht mehr existiert. 
bogen von höchstens dem k-OW $(k+1)$. Dementsprechend bedienen wir uns z 1 seinem Beweise des nachstehenden Hilfssatzes (Nr. 3.5.1.), welcher dem Hilfssatz in Nr. 3.3.1. entspricht:

3.5.1. Vor. Der Grundbogen $\mathbf{B}$ besitze den $\mathrm{k}-\mathrm{OW}(\mathrm{k}+1)$ mit $\mathrm{k} \geq 1$. Ferner seien $\mathbf{S}^{\prime}$ und $\mathrm{S}^{\prime \prime}$ avei $\mathbf{k}-$ Scheitel (also innere Punkte) von $\mathbf{B}$ und es sei $\mathbf{T}$ der (bzw., wenn $\mathbf{B}$ eine Kurve ist, einer der) Teilbogen von $\mathbf{B}$ mit den Begrenzungspunkten $\mathbf{S}^{\prime}, \mathbf{S}^{\prime \prime}$. Schliesslich sei A ein unterhalb $\mathbf{T}$ gelegener Punkt von $\mathbf{B}$ und $\mathbf{k}^{*}$ das System der OCh durch A.

$\mathrm{Beh}$. Auf dem abgeschlossenen Teilbogen $\mathbf{T}$ liegt mindestens ein $\mathrm{k}^{*}$-Scheitel.

Bew. Die Beh. ergibt sich $\left({ }^{37}\right)$ aus dem Kontraktionssatz.

3.5.2. Jeder Grundbogen $\mathbf{B}$ vom $\mathrm{k}-\mathrm{OW}(\mathrm{k}+1)$ enthält nicht mehr als $(\mathrm{k}+1) \mathbf{k}-$ Scheitel.

$\mathrm{Bew}$. Vollständige Induktion.

(1) Zunächst sei $k=1$ (bei beliebig, aber endlich vielen Grundpunkten). Es seien ferner $S_{j}, j=1,2,3$, drei $\mathbf{k - S c h e i t e l ~ i n ~ n a t u ̈ r l i c h e r ~ A n o r d n u n g ~ a u f ~}$ B, wobei $S_{1}$ unterhalb $S_{2}$ (und $S_{2}$ unterhalb $S_{3}$ ) liegt. Wegen $k+1=2$ ist mit jedem Punkt von $\mathbf{B}$ noch höchstens ein Punkt von $\mathbf{B}$ verbunden. Da $s_{j}$ ein $\mathbf{k}$-Soheitel ist, gibt es in beliebig kleiner Umgebung $\mathbf{U}_{j}$ von $S_{f}$ auf $\mathbf{B}$ zwei mit einander verbundene Punkte $P_{j}^{\prime}, P_{j}^{\prime \prime}, j=1,2,3$; o.B.d.A. können wir dabei annehmen, dass $P_{j}^{\prime}$ unterhalb $P_{j}^{\prime \prime}$ liegt und dass die $\mathbf{U}_{j}$ paarweise fremd sind, sodass $U_{1}$ unterhalb $U_{2}$ und $U_{2}$ unterhalb $U_{3}$ liegt. Wir schreiben $Q=P_{1}$ und $R=P_{1}^{\prime \prime}$. Nun lassen wir $R$ monoton nach oben rücken; dann rückt $Q$ monoton nach unten. Dabei können, selbst nach dem etwaigen Verlust von $Q$ (über den $\left({ }^{38}\right)$ Anfangspunkt $A$ von $\mathbf{B}$ hinweg), innerhalb B keine Gewinne stattfinden, weil dabei (mindestens) zwei nene, mit $R$ verbundene Punkte auf B auftreten würden. Andererseits muss $Q$ nach unten hin den Bogen $\mathbf{B}$ über $A$ hinweg verlassen bevor $R$ mit $P_{a}^{\prime}$ zusammenrückt; denn sonst wäre mit $R=P_{2}^{\prime}$ ausser $P_{2}^{\prime \prime}$ noch $Q$ verbunden. Nachdem $Q$ über $A$ hinweg nach unten hin verloren worden ist und bevor $R$ mit $P_{z}^{\prime}$ zusammenrückt, muss ferner genau ein Punkt $T$ gewonnen werden und zwar, weil dies nicht innerhalb vi $\mathbf{B}$ und nicht unterhalb $R$ möglich ist, von oben her über den Endpunkt $\left({ }^{38}\right)$ $E$ von $B$ hinweg. Sóbald $R$ mit $P_{2}^{\prime}$ zusammengerickt ist, muss $T$ monoton von $E$ nach $P_{2}^{\prime \prime}$ gelangt sein. Somit müsste $T$ vorher (monoton) jeden Punkt von $\mathbf{U}_{3}$ passieren, also $T$ etwa mit $P_{3}^{\prime \prime}$ zusammenrücken. Für $T=P_{3}^{\prime \prime}$ wäre aber $T$, ausser mit $R$, noch mit $P_{3}^{\prime}$ verbunden; da indes $P_{3}^{\prime}$ oberhalb $\mathrm{U}_{2}$

(37) Vgl. a. a. O. (5), Nr. 4.2. Übrigens gilt Nr. 3.5.1. nnabhingig von Vor. IVa., Nr. 2.1.

(38) Ist $B$ eine Kurve, so kann man als Anfangspunkt $A$ einen beliebigen, unterhalb $S_{i}$ und oberhalb $S_{3}$ gelegonen Punkt whlen; als Fndpunkt $E$ kann ebenfalls $A$ genommen werden. 
liegt und infolgedessen von dem, noch unterhalb $P_{2}^{\prime}$ gelegonen, Punkt $R$ verschieden ist, widerspricht diese Verbundenheit von $R, P_{3}^{\prime}$ und $P_{3}^{\prime \prime}=T$ der Vor., dass B den k-OW 2 besitzt. Damit ist die Beh. für $k=1$ bewiesen.

A mmerkung. Beim Beweise wurde nur die Existenz ron drei Paaren verbundener Punkte $P_{j}^{\prime}, P_{j}^{\prime \prime}$ voriusgesetzt, die in fremden Umgebungen auf B liegen; insbesondere ist also a priori nicht voransgesetzt, dass auf $\mathbf{B} \mathrm{nur}$ beschränkt viele $\mathbf{k}$-Scheitel liegen oder dasss die $\mathbf{k}$-Scheitel von den Grundpunkten verschieden sind. Auch Vor. IVa. wurde nicht benutzt.

(2) Induktionsannahme. Die Beh. sei schon für alle $k$ mit $1 \leq k \leq k^{\prime}$ bewiesen. Angenommen, es sei $k^{\prime \prime}=k^{\prime}+1$ die Grundzahl eines OCh-Systems $\mathbf{k}^{\prime \prime}$. Ferner besitze $\mathbf{B}$ den $\mathbf{k}^{\prime \prime}-0 \mathrm{~W}\left(k^{\prime \prime}+1\right)$. Ist höchstens ein $\mathbf{k}^{\prime \prime}$-Scheitel vorhanden, so ist die Beh. richtig, wegen $1<k^{\prime \prime}$. Es enthalte $\mathbf{B}$ in Innern (mindestens) die $k^{\prime \prime}$-Scheitel $S_{1}, \ldots, S_{2 q}$ in natiorlicher Anordnung, wobei $S_{1}$ unterhalb $S_{2}$ liegt und wobei $1 \leq q$; die Annahme der Existenz von $-q \mathbf{k}^{\prime \prime}-$ Scheiteln , widerspricht nicht der Behauptung, da jedenfalls für $q=1$ gilt: $2 q \leq k^{\prime}+1<k^{\prime \prime}+1$. Weiter sei $A$ ein, von den $\mathbf{k}^{\prime \prime}$-Grundpunkten verschiedener, Punkt von $\mathbf{B}$ untorhalb $S_{i}$ (und oberhalb $S_{: q}$, falls $\mathbf{B}$ eine Kurve sein sollte); das System aller OCh aus $\mathbf{k}^{\prime \prime}$, die den Punkt $A$ enthalten, sei $\mathbf{k}^{\prime}$. L's ist $\mathbf{k}^{\prime}$ ein OCh-System mit der Grundzahl $k$. Zufolge Nr. 3. J.1, existieren (mindestens) $q$ k'-Scheitel, nämlich jeweils einer in dem abgeschlossenen Teilbogen mit. $S_{2 \tau-1}$ bzw. $S_{2 \tau}$ als Anfangs- bzw. Endpunkt, $\tau=1, \ldots, q$. Nach Induktionsannahme ist aber $q \leq k^{\prime}+1$; denn $\mathbf{B}$ besitzt (höchstens) den $\mathrm{k}^{\prime}-0 \mathrm{~W} k^{\prime \prime}=k^{\prime}+1$. Mithin besitzt B nur beschränkt viele $\mathbf{k}^{\prime \prime}-$ Scheitel, nämlich nicht mehr als $2\left(k^{\prime}+1\right)+1$. Nunmehr können wir die Beh. (dieser Nr. 3.5.2.) für $k=k^{\prime \prime}$ selbst beweisen. Dazu wählen wir $A$ derart, dass jede der (beschränkt vielen) $\mathbf{k}^{\prime \prime}$-Scheitelparatingenten an $\mathbf{B}$ fremd ist zu $A$, dass also die $\mathbf{k}^{\prime}$-Scheitel von den $\mathbf{k}^{\prime \prime}$-Scheiteln verschieden sind und mithin insbesondere $A$ selbst kein $\mathbf{k}^{\prime}$-Scheitel ist (vgl. die auf $S_{1}$ bezüglichen Bemerkungen im Nr. 3.4.2., Bew., Induktionsannahme); hierbei wird Nr. 2.1., IVa., wesentlich benutzt. Sind dann $S_{1}, \ldots, S_{p}$ die $\mathbf{k}^{\prime \prime}$-Scheitel von B, so enthält jeder Bogen mit $S_{\rho}$ als Anfangs- und $S_{\rho+1}$ als Endpunkt $(\rho=1, \ldots, p-1)$ mindestens einen $\mathbf{k}^{\prime}-$ Scheitel in Innern (gemäss Nr. 3.5.1.), sodass die Anzahl der $\mathbf{k}^{\prime}$-Scheitel mindestens $(p-1)$ ist. Nach Induktionsannahme ist aber die Anzahl der $\mathbf{k}^{\prime}$-Scheitel höchstens gleich $\left(k^{\prime}+1\right)$. Mithin ist $p-1 \leq k^{\prime}+1$ oder $p \leq k^{\prime}+2=k^{\prime \prime}+1$. Damit ist der Induktionsschluss fertig. Er gilt insbesondere auch für den Fall einer Grundkurve.

3.6. Beweis von Satz (B) (5) in Nr. 2.2.

Es gelten die Vor. I.-IV. (Nr. 2.1.).

3.6.1. Enthäll der Grundbogen B (in seinem Innern) nur isolierte k-Scheitel, so sind je zwei $\mathbf{k}-S c h e i t e l \mathbf{S}^{\prime}, \mathrm{S}^{\prime \prime}$ von $\mathbf{B}$ gleich-oder ungleicharig, je nachdem 
die Gesamta lhl der $\mathbf{k}$-Grundpunkte und $\mathbf{k}$-Scheitel auf $\mathbf{B}$, durch die $\mathrm{S}^{\prime}$ und $\mathrm{S}^{\prime}$ auf $\mathbf{B}$ von einander getrennt verden, ungerade oder gerade ist.

Zur Definition del Gesantzahl der k-Grundpunkte und $\mathbf{k}-$ Scheitel, dnvel die $\mathrm{S}^{\prime}$ und $\mathrm{S}^{\prime \prime}$ von einander getrennt werden: Fält ein zwischen $S^{\prime}$ und $S^{\prime \prime}$ gelegener $\mathbf{k}$-Scheitel in einen $\mathbf{k}$-Grundpunkt, so ist der betr. Punkt sowohl als k-Grundpunkt wie als k-Scheitei zu zahlen; fällt $S^{\prime}$ in einen k-Grund. punkt, so ișt $S^{\prime \prime}$ als zwischen $S^{\prime}$ und $S^{\prime \prime}$ gelegener k-Grundpunkt zu zählen.

Bew. Gemäss Nr. 3.2.4. ist jede hinreichend kleine, einseitige Umgebung von $S^{\prime}$ oder $S^{\prime \prime}$ auf $\mathbf{B}$ vom $\mathbf{k - O W} k$; insbesondere liegen $S^{\prime}$ und $S^{\prime \prime}$ im Innern von B. Es liege etwa $S^{\prime \prime}$ oberhalb $S^{\prime}$ und es sei $S^{\prime}$ bzw. $S^{\prime \prime}$ Anfangsbzw. Endpunkt des Teilbogens $\mathbf{S}$ von B. Da die k-Scheitel isoliert auf $\mathbf{B}$ liegen sollen, genügt es, den Fall zu betrachten, dass im Innern von $\mathbf{S}$ keine weiteren k-Scheitel liegen, und zu zeigen: $S^{\prime}$ und $S^{\prime \prime}$ sind gleich-bzw. ungleichartig, je nachdem $S^{\prime}$ von $S^{\prime \prime}$ auf $\mathbf{S}$ durch eine ungerade oder gerade Anzahl von Grundpunkten getrennt werden; zu diesen Grundpunkten ist $S^{\prime}$ selbst zu zahlen, falls $S^{\prime}$ mit einem Grundpunkt zusammenfallt (Beachte, dass $S^{\prime}$ unterhalb $S^{\prime \prime}$ liegen sollte). Es sei nun $V^{\prime}$ bzw. $V^{\prime \prime}$ eine untere bzw. obere Umgebung von $S^{\prime}$ bzw. $S^{\prime \prime}$ je vom k-OW $k$. Für jedes r-tupel mit $k+1 \leq r$, dessen zugehöriger Teilbogen $\mathbf{R}$ in $\tilde{\mathbf{S}}=\mathbf{V}^{\prime}+\mathbf{S}+\mathbf{V}^{\prime \prime}$ enthalten ist, muss $\mathbf{R}$ in seinem Innern entweder $S^{\prime}$ oder $S^{\prime \prime}$ oder beide enthalten, weil nämlich $\mathbf{S}$ den k-OW $k$ besitzt. Ws seien $\mathbf{U}^{\prime}$ bzw. $\mathbf{U}^{\prime \prime}$ (zweiseitige) Umgebungen von $S^{\prime}$ bzw. von $S^{\prime \prime}$, die in $\tilde{\boldsymbol{S}}$ enthalten und fremd sind sowie keinen der (endlich vielen) Grundpunkte enthalten ansser höchstens $S^{\prime \prime}$ und $S^{\prime \prime}$. Es existicrt dann ein in $\mathbf{U}^{\prime}$ bzw. $\mathbf{U}^{\prime \prime}$ enthaltenes $(k+1)$-tupel $\gamma^{\prime}=\left(S_{1}^{\prime}, \ldots, S_{k+1}^{\prime \prime}\right)$ byw. $\gamma^{\prime \prime}=\left(S_{t}^{\prime \prime}, \ldots, S^{\prime \prime}{ }_{k+1}\right)$; dabei muss $\gamma^{\prime}$ bzw. $\gamma^{\prime \prime}$ Punkte sowohl unter-als oberhalb $S^{\prime \prime}$ bzw. $S^{\prime \prime}$ enthalten, weil andernfalls z.B. $U^{\prime}$ einon unter-oder oberhalb $S^{\prime}$ gelo. genen k-Scheitel enthalten würde.

Wir ändern nun $\gamma^{\prime}$ stetig derart, dass sich $S_{1}^{\prime}$ nach unten und $S_{k+1}^{\prime}$ nach oben bewegt, whhrend $S_{2}{ }^{\prime}, \ldots, S_{k}{ }^{\prime}$ festgehalten werdon. Im Verlaufe dieser Änderung kann solange kein Punkt innerhalb $S^{\prime}=V^{\prime}+S$ gewonnen werden, als $S^{\prime \prime}{ }_{k+1}$ unterhalb $S^{\prime \prime}$ bleibt. In der Tat: Jedenfalls ist dies richtig, solange $S_{i}^{\prime}$ nach unten hin nicht aus $S^{\prime}$ (über den Anfongspunkt $V^{\prime}$ von $V^{\prime}$ hinweg) hinausrückt; denn $\mathbf{S}^{\prime}$ besitzt cen $\mathbf{k}-\mathrm{OW}(k+1)(\mathrm{Nr} .3 .3 .1$, (b) (II)). Aber anch nachdem $S_{1}$ über $V^{\prime}$ hinweg den Bogen $\mathbf{S}^{\prime}$ verlassen haben sollte, kann in $\mathbf{S}^{\prime}$ kein Gewinn stattfinden. Denn einerseits sind Gewinne in $\mathbf{S}^{\prime}$ unterhalb $S_{k+1}^{\prime}$ unmöglich, weil der von $V^{\prime}$ und $S_{k+1}^{\prime}$ begrenzte Teilbogen von $\mathbf{S}^{\prime}$ stäindig wächst. Andererseits sind Gewinne in $\mathbf{S}^{\prime}$ oberhalb $S_{k+1}^{\prime}$ unmöglich; würde namlich ein Schnittpunkt $S_{k+2}^{\prime}$ innerhalb $\mathbf{S}^{\prime}$ and oberhalb $S_{k+1}^{\prime}$ auftreten, so existierte ein $(k+1)$-tupel $\gamma_{2}^{\prime}$ in $S^{\prime}$ mit $S_{2}^{\prime}$ als Anfangspunkt; es werden aber $S_{1}^{\prime}$ und $S_{2}^{\prime}$ entweder durch einen Grundpunkt (numlich durch $S^{\prime \prime}$ ) oder durch keinen Grundpunkt auf $\mathbf{U}^{\prime}$ getrennt, je nachdem entweder $S^{\prime \prime}$ sowohl in einen Grundpunkt fallt, als zwischen $s_{1}^{\prime}$ ind $S_{2}^{\prime}$ liegt oder nicht beides gleichzeitig 
der Fall ist; daher würden, weil $S_{1}{ }^{\prime}$ und $S_{2}{ }^{\prime}$ in $\gamma^{\prime}$ unmittelbar aufeinanderfolgen, je nachdem $\gamma^{\prime}$ und $\gamma_{2}^{\prime}$ gleichartig oder ungleichartig sein und es würden dann gemäss N. 3.3.1., (a), innerhalb $\mathbf{S}^{\prime}$ zwei verschiedene $\mathbf{k}$-Scheitel existieren im Widerspruch mit der Definition von $\mathbf{S}^{\prime}$. Wir können also durch unsere Änderung jedenfalls $S_{k+1}^{\prime}$.in eine in $\mathbf{U}^{\prime \prime}$ enthaltene untere Umgebung $\mathbf{W}^{\prime \prime}$ von $S_{1}^{\prime \prime}$ bringen, ohne dass in $\mathbf{S}^{\prime}$ Gewinne stattfinden; die Endlage von $S_{k+1}^{\prime}$ sei $T_{k+1}$. (Han beachte, dass $S_{1}^{\prime \prime}$ unterhalb $S^{\prime \prime}$ liegen muss, weil andernfalls in $\mathbf{U}^{\prime \prime}$ oberhalb von $S^{\prime \prime}$ noch ein k-Scheitel liegen würde).

Durch Wiederholung dieser Schlüsse (jetzt bei festen $S_{2}{ }^{\prime}, \ldots, S_{k-1}^{\prime}, T_{k+1}$; usw.) ergibt sich (gleichgültig ob $S_{1}^{\prime}$ den Punkt $V^{\prime}$ nach unten hin überschritten hat oder nioht) die Existenz eines in $W^{\prime \prime}$ enthaltenen, also unterhalb $S_{1}$ "gelegenen $k$-tupels $\tau=\left(T_{2}, \ldots, T_{h+-1}\right)$, von dem folgendes behauptet werden kann: Es ist $\tau$ gleich-oder ungleichartig mit $\gamma^{\prime}$, je nachdem $S^{\prime}$ und $S^{\prime \prime}$ auf $\mathbf{S}$ durch eine nngerade oder gerade Anzahl von Grundpunkten getrennt werden (dabei ist $S^{\prime}$ unter diese Grundpunkte mit einzurechnen, falls $S^{\prime}$ in einen Grundpunkt fallt). In der Tat: Ist zunächst $S^{\prime}$ kein Grundpunkt, dann ist stets $\gamma^{\prime}$ ungleichartig mit $\tau^{\prime}=\left(S_{2}^{\prime}, \ldots, S_{k+1}^{\prime}\right)$; je nachdem dann eine gerade oder ungerade Anzahl Grundpunkte zwischen $S^{\prime}$ und $S^{\prime \prime}$ liegt, sind $\tau^{\prime}$ und $\tau$ gleich-oder ungleichartig, also $\gamma^{\prime}$ und $\tau$ ungleich-oder gleichartig, wie behauptet. Es sei nun $S^{\prime}$ ein Grundpunkt; liegt $S^{\prime}$ nicht zwischen $S_{1}^{\prime}$ und $S_{2}^{\prime}$, so schliesst man wie eben, wobei $S^{\prime}$ als Grundpunkt zwischen $S^{\prime}$ und $S^{\prime \prime}$ zu zahlen ist; liegt aber $S^{\prime}$ zwischen $S_{1}^{\prime}$ und $S_{2}^{\prime}$ so, ist $\gamma^{\prime}$ gleichartig mit $\tau^{\prime}$ und dieses gleich-bzw. ungleichartig mit $\tau$ je nachdem zwischen $S^{\prime}$ und $S^{\prime \prime}$ eine gerade oder ungerade Anzahl von Grundpunkten liegt, wobei jetzt $S^{\prime}$ nicht mitzahlt. Daraus folgt wieder die Beh. betr. $\gamma^{\prime}$ und $\tau$. - Wir unterscheiden für den weiteren Verlauf des Beweises folgende Fälle: Entweder (Fall A.) ist in $\mathbf{U}^{\prime \prime}$ ein (oberhalb $S^{\prime}$, gelegener) Schnittpunkt $T_{k+2}$ mit $\tau$ verbunden (mehr als ein solcher Schnitt. punkt kann nicht existieren); es sind alle in $\mathbf{U}^{\prime \prime}$ gelegenen $(k+1)$-tupel gleichartig mit $\tau$, insbesondere also auch $\gamma^{\prime}$ (gemäss Nr. 3.3.3.), da $\mathbf{U}^{\prime \prime}$ keine Grundpunkte enthalten soll abgesehen höchstens von $S^{\prime \prime}$. Oder (Fall B.) es ist mit $\tau$ kein Schnittpunkt in $\mathbf{U}^{\prime \prime}$ verbunden. Dann halten wir $T_{2}, \ldots, T_{k}$ fest und lassen $T_{k+1}$ monoton nach oben hin sich bewegen entweder (Fall $\mathrm{B}^{\prime}$.) bis in $\mathbf{U}^{\prime \prime}$ von oben her ein neuer, mit $\tau$ verbundener Schnittpunkt uber den Endpunkt von $\mathbf{U}^{\prime \prime}$ nach $\mathbf{U}^{\prime \prime}$ hereinruckt, womit wir auf den Fall $A$. zurückkommen; oder $\left(\right.$ Fall $\mathrm{B}^{\prime \prime}$.) bis $T_{k+1}$ mit $S_{k}^{\prime \prime}$ zusammenrückt; in diesem Falle $\mathrm{B}^{\prime \prime}$. ist wieder $\mathrm{zu}$ beachten, dass unterhalb $T_{k+1}$ in $\mathbf{S}^{\prime}$ keine Gewinne stattfinden können, weil derjenige Teilbogen von $\tilde{\mathbf{S}}$ wächst, der $S_{1}^{\prime}$ oder, falls $S_{1}^{\prime}$ schon verloren ging, $V^{\prime}$ als Anfangs-und $T_{k+1}$ als Endpunkt besitzt. Hat man nun $T_{k+1}=S_{k}$ " erreicht, so wiederhole man das Verfahren mit dex Modifikation, dass man jetzt bei festen $T_{2}, \ldots, T_{k-1}, S_{k}{ }^{\prime \prime}$ den Schnittpunkt $T_{k}$ nach oben hin gegen $S^{\prime \prime}{ }_{k+1}$ sich bewegen lässt. Entweder tritt einmal Fall A. oder Fall B. ein oder das Verfahren lässt sich erneut wiederholen. Würde dabei immer Fall 
$\mathrm{B}^{\prime \prime}$. eintretén, so liessen sich der Reihe nach $T_{j+1}$ und $S_{j}^{\prime \prime}$ zur Deckung bringen, $j=k, k-1, \ldots, 1$. Dann aber wäre $S^{\prime \prime}{ }_{k+1}$ mit dem $k$-tupel $\left(T_{2}=S_{1}^{\prime \prime}, \ldots, T_{k+1}=S_{k}{ }^{\prime \prime}\right)$ verbunden und folglich mïsste, schon bevor $T_{j+1}$ und $S_{j}^{\prime \prime}$ für alle $j$ zur Deckung gebracht ist, einmal Fall A. oder B'. eingetreten sein; entgegen der Annahme. Damit erweist sich aber $\tau$ als in .jedem Falle gleichartig mit $\gamma^{\prime \prime}$. Daraus folgt die Beh.

3.6.2. Besitat die Grundkurve $\mathbf{C}$ nur endlich viele $\mathbf{k}-$ Scheitel, so ist die Gesamtzahl der $\mathbf{k}$-Scheitol und der $\mathbf{k}$-Grundpunkte gerade; dabei ist jeder k-Scheitel, der mit einem k-Grundpunkt zusammenfällt, in der Gesamtzahl zweifach zu zählen, nämlich einmal als $\mathbf{k - S c h e i t e l ~ u n d ~ d a n n ~ a l s ~} \mathrm{k}-$ Grundpunkt.

Bew. Sind $S_{1}, \ldots, S_{g}$ die k-Scheitel von $\mathbf{C}$ in natürlicher Anordnung, sind also $S_{q+1}=S_{1}$ und $S_{q}$ unmittelbar benachbart auf $\mathbf{C}$, ist ferner $t_{\rho}$ die im Sinne des Satzes von Nr. 3.6.1. berechnete Anzahl der Grundpunkte zwischen $S_{p}$ und $S_{p+1}, \rho=1, \ldots, q$, so sind $S_{p}$ und $S_{\rho+1}$ gleich-oder ungleichartig je nachdem $s_{p} \equiv t_{p}+1 \equiv 0$ oder $\equiv 1(\bmod 2)$ ist. Wegen $S_{q+1}=S_{1}$ muss daher die Anzahl der $s_{f}$ mit $s_{q} \equiv 1$ gerade sein, also $s_{1}+\ldots+s_{q}=\left(t_{1}+\ldots+t_{q}\right)+q \equiv 0$ woraus die Beh. folgt. 